MATHEMATICS OF COMPUTATION

Volume 71, Number 240, Pages 1339-1370

S 0025-5718(01)01359-X

Article electronically published on June 14, 2001

\title{
EFFECTS OF UNCERTAINTIES IN THE DOMAIN ON THE SOLUTION OF NEUMANN BOUNDARY VALUE PROBLEMS IN TWO SPATIAL DIMENSIONS
}

\author{
IVO BABUŠKA AND JAN CHLEBOUN
}

\begin{abstract}
An essential part of any boundary value problem is the domain on which the problem is defined. The domain is often given by scanning or another digital image technique with limited resolution. This leads to significant uncertainty in the domain definition. The paper focuses on the impact of the uncertainty in the domain on the Neumann boundary value problem (NBVP). It studies a scalar NBVP defined on a sequence of domains. The sequence is supposed to converge in the set sense to a limit domain. Then the respective sequence of NBVP solutions is examined. First, it is shown that the classical variational formulation is not suitable for this type of problem as even a simple NBVP on a disk approximated by a pixel domain differs much from the solution on the original disk with smooth boundary. A new definition of the NBVP is introduced to avoid this difficulty by means of reformulated natural boundary conditions. Then the convergence of solutions of the NBVP is demonstrated. The uniqueness of the limit solution, however, depends on the stability property of the limit domain. Finally, estimates of the difference between two NBVP solutions on two different but close domains are given.
\end{abstract}

\section{INTRODUCTION}

The analysis presented in this paper has been motivated by the discrepancy between the shape of a real body and its computer description (called geometrical model or briefly model).

Any real-life data contain some uncertainty due to measurements and simplifications. It is common to represent a real-life body by the geometrical model and to neglect the fact that the model is obtained by postprocessing the raw data from scanning, for example. Instead of the true body, the model is used for solving partial differential equations. However, natural questions arise: Are we authorized to choose a particular geometrical model as the representative of the body? Should we take a whole family of models into consideration? Can we get rid of assumptions we added to the raw data by a particular postprocessing method? How does

Received by the editor August 5, 1999 and, in revised form, October 13, 2000.

2000 Mathematics Subject Classification. Primary 65N99, 65N12, 35J25.

Key words and phrases. Elliptic equation, Neumann boundary condition, uncertainty.

The research of the first author was funded partially by the National Science Foundation under the grant NSF-Czech Rep. INT-9724783 and NSF DMS-9802367.

Partial support for the second author, coming from the Ministry of Education of the Czech Republic through grant ME..148(1998) as well as from the Grant Agency of the Czech Republic through grant 201/98/0528, is appreciated. 


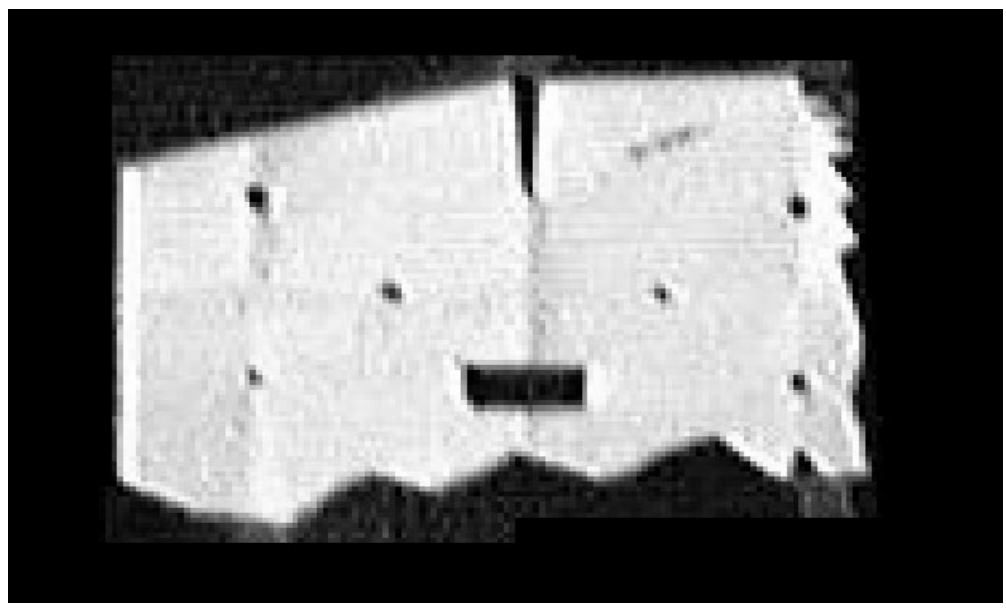

Figure 1. Original digital image

the discrepancy between the body and its geometrical model influence a boundary value problem (BVP) we wish to solve?

These questions are closely related to the problem of model validation (see $[\mathbf{R}]$ ). Though natural, they are not mathematically analyzed.

Let us restrict ourselves to the problems stemming from digital imaging and image processing. A digital image of a real-life body bears some inaccuracy, the source of which is both the scanning and pixel-limited resolution. Setting the scanning aside and concentrating only on the digital image, we still face uncertainty regarding the boundary of a digital domain. We can color black the pixels lying fully inside the domain and white the fully outside pixels. Then a boundary layer can remain. A layer of gray pixels indicates that these pixels are partly "in" and partly "out". Thus the boundary is not known exactly and any approximation or smoothening can be problematic. (We refer to [BPHN for more about data smoothening.)

Example 1.1. Figure 1 shows a rather fuzzy digital image. Since pixels are rough, contrast low, and data noisy, we can hardly define the boundary separating the supposedly white domain and a supposedly black background. The image can be postprocessed in various ways.

We can set a threshold brightness value to suppress the gray color and to strictly define sets above (white pixels) and below (black pixels) the threshold (see Figure 2 (left)).

We could also apply a sophisticated algorithm to guess and approximate the boundary by a piecewise polynomial curve (see Figure 2 (right)). These algorithms use various heuristic approaches based on additional assumptions usually not justified in relation to the solved problem (see $[\mathrm{BCL}],[\mathrm{BPHN}]$ ).

The reader probably agrees that low quality images pose difficulties. But even analysis based on high quality images can lead to incorrect results.

For example, the common formulation of the Neumann boundary condition could be misleading if an uncertain boundary is taken into account without realizing 

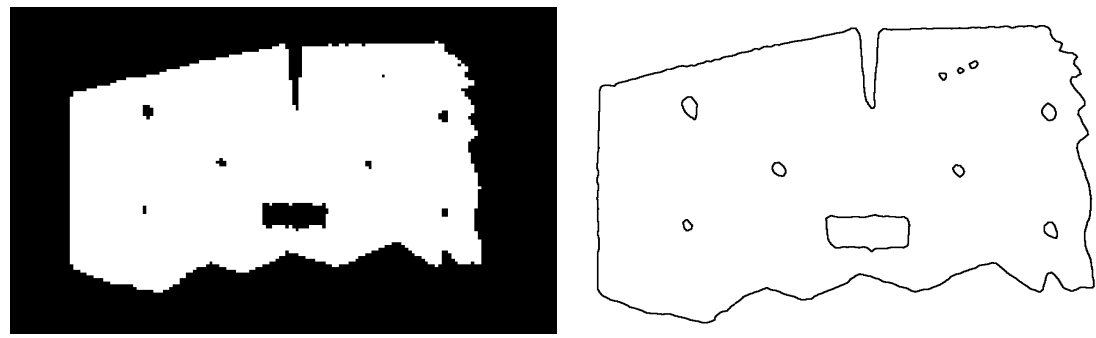

Figure 2. Postprocessing: Black and white image (left), smoothed boundary (right)

further consequences. We illustrate this in the following example showing what happens if a naive approximation of the Neumann problem is used.

Example 1.2. Let $\Omega$ be a disc with the center at the origin and the unit radius. We consider the weak solution $u \in H^{1}(\Omega)$ of the Neumann problem $-\Delta u+u=0$ in $\Omega$ and $\partial u / \partial \nu=1$ on $\partial \Omega, \nu$ is the outward normal unit vector. In detail,

$$
\int_{\Omega}(\nabla u \cdot \nabla v+u v) \mathrm{d} x=\int_{\partial \Omega} v \mathrm{~d} s \quad \forall v \in H^{1}(\Omega) .
$$

To fix ideas, let us consider the physical interpretation of (1.1). The disk $\Omega$ represents a thin cylinder after dimensional reduction. The part of the cylinder boundary represented by $\partial \Omega$ is in contact with a heat producing coil. The heat enters $\Omega$ through $\partial \Omega$ with the flux equal to 1 . In this setting, (1.1) can be viewed as the equation modeling a time independent heat flow.

Let us suppose we have an infinite sequence of digital images of $\Omega$ at our disposal and assume that $\Omega$ is approximated by a sequence $\left\{\Omega_{n}\right\}_{n=1}^{\infty}$ of pixel-formed domains $\Omega_{n}, \Omega_{n} \subset \Omega_{n+1} \subset \Omega \subset \mathbb{R}^{2}, \Omega_{n} \nearrow \Omega$, i.e., the larger the $n$, the tinier the pixels. Then it seems to be natural to approximate (1.1) by

$$
\int_{\Omega_{n}}\left(\nabla u_{n} \cdot \nabla v+u_{n} v\right) \mathrm{d} x=\int_{\partial \Omega_{n}} v \mathrm{~d} s \quad \forall v \in H^{1}\left(\Omega_{n}\right)
$$

and seek the respective solution $u_{n} \in H^{1}\left(\Omega_{n}\right)$. Let us mention that the pixels are sometimes used as a natural mesh for the finite element method.

Will $u_{n}$ tend to $u$ if $\Omega_{n} \rightarrow \Omega$ ? The answer is "no".

A simple reason is that the length of $\partial \Omega_{n}$ does not converge to the length of $\partial \Omega$. Indeed, if the diameter of a pixel domain $\Omega_{n}$ is close to 2 , then the sum of all vertical and horizontal boundary segments of $\Omega_{n}$ is close to 8 . Denoting $l_{n}$ the length of $\partial \Omega_{n}$, we thus get $\lim _{n \rightarrow \infty} l_{n}=8$.

Let us consider $v=1$ in (1.1) and (1.2). Then $\nabla v=0$ and we get

$$
\begin{aligned}
& (\text { meas } \Omega)^{1 / 2}\left\|u-u_{n}\right\|_{L^{2}\left(\Omega_{n}\right)}+\int_{\Omega \backslash \Omega_{n}}|u| \mathrm{d} x \\
& \quad \geq \int_{\Omega_{n}}\left(u_{n}-u\right) \mathrm{d} x-\int_{\Omega \backslash \Omega_{n}} u \mathrm{~d} x=\int_{\Omega_{n}} u_{n} \mathrm{~d} x-\int_{\Omega} u \mathrm{~d} x=\int_{\partial \Omega_{n}} \mathrm{~d} s-\int_{\partial \Omega} \mathrm{d} s .
\end{aligned}
$$

Consequently,

$$
\liminf _{n \rightarrow \infty}\left\|u-u_{n}\right\|_{L^{2}\left(\Omega_{n}\right)} \geq(8-2 \pi) / \sqrt{\pi}
$$


It is possible to prove that solutions $u_{n}$ converge to a function $u_{0}$ solving a Neumann problem on $\Omega$ but with a different boundary condition, i.e., $\partial u_{0} / \partial \nu=g_{0} \neq 1$ on $\partial \Omega$.

Let us compare three problems:

$$
\begin{array}{llll}
-\Delta u_{a}+u_{a}=0 & \text { in } \Omega, & \frac{\partial u_{a}}{\partial \nu}=1 & \text { on } \partial \Omega ; \\
-\Delta u_{b}+u_{b}=1 & \text { in } \Omega, & \frac{\partial u_{b}}{\partial \nu}=0 & \text { on } \partial \Omega ; \\
-\Delta u_{c}+u_{c}=1 & \text { in } \Omega, & \frac{\partial u_{c}}{\partial \nu}=1 & \text { on } \partial \Omega .
\end{array}
$$

Problem (1.4) coincides with (1.1). If (1.5) and its pixel approximation were considered instead of (1.1) and (1.2), respectively, approximate solutions $u_{b_{n}}$ would perfectly converge to $u_{b}$. On the other hand, (1.6) encounters difficulties similar to those in Example 1.2. This indicates that the toothy boundary and its length are not the only culprits. We must also take into account the type of the boundary condition.

Let us pay attention to the right-hand side of (1.2). We were wrong setting $\partial u_{n} / \partial \nu=1$. Is it possible to replace the classical formulation so that the abovementioned difficulty will be avoided?

Let us suppose that we know the heat produced by the coil and entering $\Omega$ through $\partial \Omega$. We can get it with the aid of a voltameter and an amperemeter. The same amount enters each $\Omega_{n}$. We know the length of $\partial \Omega_{n}$ and thus we are able to calculate an averaged heat flux along $\partial \Omega_{n}$. Obviously, the longer $\Omega_{n}$, the lower average we get.

In general, the right-hand side of (1.1) and (1.2) has the form $\int_{\partial \Omega} g v \mathrm{~d} s$ and $\int_{\partial \Omega_{n}} g_{n} v \mathrm{~d} s$, respectively. Functions $g_{n}$ depend on $\Omega_{n}$. Let us assume that we have two points $P_{1}, P_{2}$, and that $P_{1}, P_{2} \in \partial \Omega$ and $P_{1}, P_{2} \in \partial \Omega_{n}$. Then

$$
\int_{P_{1}}^{P_{2}} g \mathrm{~d} s_{\Omega}=\int_{P_{1}}^{P_{2}} g_{n} \mathrm{~d} s_{\Omega_{n}}
$$

because the amount of heat flowing between $P_{1}$ and $P_{2}$ does not depend on the path connecting $P_{1}$ and $P_{2}$. It resembles the Stieltjes integral. The idea is to find a function $G$ and to substitute $\int_{\partial \Omega} v \mathrm{~d} G$ for $\int_{\partial \Omega} g v \mathrm{~d} s$ and $\int_{\partial \Omega_{n}} v \mathrm{~d} G$ for $\int_{\partial \Omega_{n}} g_{n} v \mathrm{~d} s$. Pixel domains are fuzzy and their boundaries do not coincide with $\partial \Omega$; therefore $G$ should be defined at least on a strip containing $\partial \Omega$. In other words, here we are working with resultants as we often do in elasticity theory. It is not difficult to construct function $G$ on the basis of such physical considerations (see also Example 1.3 and Example 2.1).

We observe that $g=\partial G / \partial s=\operatorname{curl} G \cdot \nu$ along $\partial \Omega, \operatorname{curl} G \stackrel{\text { def }}{=}\left(\partial G / \partial x_{2},-\partial G / \partial x_{1}\right)$ and $\partial / \partial s$ stands for the tangential derivative. If $G$ is sufficiently smooth and defined on $\Omega_{n}$, then

$$
\int_{\partial \Omega_{n}} v g \mathrm{~d} s=\int_{\Omega_{n}} \operatorname{curl} G \cdot \nabla v \mathrm{~d} x \quad v \in H^{1}(\Omega) .
$$

The equality suggests that we can substitute the domain integral for the boundary one.

Remark 1.1. Going back to the physics, we can see how massively our model relies on averaging and scanning. The interaction of the coil and the body $\Omega$ is a contact 
problem. Its local features are not known. Does the coil touch $\Omega$ at all points or are there gaps?

We focus on global features expressed via the energy or energy-like norms defined on $\Omega$. Also, the scale of $\Omega$ is much greater than the scale of the local contact uncertainty. That is why we neglect the local view and prefer the global one which is based on resultants rather than on pointwise loads. This corresponds to the well-known Saint-Venant principle used in mechanics.

Using the above defined $G$, we get $\int_{\partial \Omega} \mathrm{d} G=0$, which implies $\int_{\partial \Omega} g \mathrm{~d} s=0$. As a consequence, we cannot find $G$ corresponding to problem (1.1). We will see a remedy in Section 2, however. We can simply seek two functions $G_{1}, G_{2}$, or we can reformulate the BVP.

Another natural construction of $G$ appears in the next example.

Example 1.3. The torsion of a general noncircular cylinder of cross section $\Omega$ is treated in almost any textbook on the theory of elasticity (see, e.g., $\mathrm{NH}$, Section 10.5]). In textbooks, the final goal is to derive the Dirichlet BVP for the Prandtl potential function. Before the Prandtl potential can be established, one has to study the following Neumann BVP:

$$
\Delta \varphi=0 \quad \text { in } \Omega, \quad \frac{\partial \varphi}{\partial \nu}=g \stackrel{\text { def }}{=} x_{2} \nu_{1}-x_{1} \nu_{2} \quad \text { on } \partial \Omega .
$$

Defining $G(x) \stackrel{\text { def }}{=}\left(x_{1}^{2}+x_{2}^{2}\right) / 2, x=\left(x_{1}, x_{2}\right) \in \mathbb{R}^{2}$, we get $g=\operatorname{curl} G \cdot \nu$ along $\partial \Omega$. The weak formulation of (1.7) reads: Find $\varphi \in H^{1}(\Omega)$, up to a constant, such that

$$
\int_{\Omega} \nabla \varphi \cdot \nabla v \mathrm{~d} x=\int_{\Omega} \operatorname{curl} G \cdot \nabla v \mathrm{~d} x \quad \forall v \in H^{1}(\Omega),
$$

where we used the Green theorem to get rid of the boundary integral. Let us notice that $G$ is unique (up to a constant), defined in $\mathbb{R}^{2}$, and is given in a straightforward way by the boundary condition in (1.7) because the condition applies to any admissible domain $\Omega$.

Unlike (1.7), where $g$ cannot be defined if $\partial \Omega$ is fuzzy, $G$ is defined irrespectively of the fuzziness. As the fuzziness is limited to a thin boundary layer, (1.8) enables us to assess the effect that the uncertain boundary has on the solution $\varphi$ (see Section 4).

We can draw a general conclusion from Examples 1.1 and 1.2. It is necessary to extend the notion of well-posed problems to a continuous dependency of the BVP solution on the domain of definition, i.e., to generalize Hadamard's ideas (see $[\mathrm{H}]$ ).

We already saw (Example 1.2) that a sequence of pixel-based problems can have little in common with the seemingly natural limit BVP.

Though we do not have an infinite sequence of pixel domains in practice, we should ask what the limit solution could be if our digital camera delivered a sequence of domains $\Omega_{n}$ converging to a limit $\Omega$. It means that the stability of the domain $\Omega$ with respect to the Neumann BVP comes in the forefront.

The stability issue was studied in [B1], [B2] for rather general Dirichlet problems and the Neumann BPV with the homogeneous boundary condition. Roughly speaking, if $\Omega$ is stable, then solutions of equations on $\Omega_{n}$ converge to the function which solves a natural limit problem on $\Omega$, i.e., the solution is not sensitive to the approximation of the domain. It is known that if $\partial \Omega$ is Lipschitz, then $\Omega$ is stable (see also Section 3). 
If $\Omega$ is unstable, then, in general, the limit depends on the sequence $\left\{\Omega_{n}\right\}_{n=1}^{\infty}$. This means that the solution is significantly influenced by the uncertainty in the approximation of the boundary $\partial \Omega$.

The circle is stable with respect to the classical Neumann BVP with the homogeneous boundary condition and is unstable for nonhomogeneous boundary conditions (cf. (1.1)-(1.2)). Nevertheless, we can reformulate the nonhomogeneous boundary conditions so that the stability (i.e., the nonsensitivity of the solution to the boundary) occurs (see Section 2).

In real life, we are limited to a few digital images of the true body so we do not know the limit domain $\Omega$. We only assume that the images give us a lower and an upper bound of (possibly unstable) $\Omega$; namely, two domains $\Omega_{\text {low }}$ and $\Omega_{\text {up }}$ such that $\Omega_{\text {low }} \subset \Omega \subset \Omega_{\text {up }}$.

Our goal is to show that the Neumann problem for a nonhomogeneous boundary condition can be reformulated so that it is stable in contrast to the classical (weak) formulation, and to assess the solution of the Neumann BVP on the unknown domain $\Omega$ by means of the solutions of the Neumann BVP on $\Omega_{\text {low }}$ and $\Omega_{\text {up }}$.

This paper is organized as follows. Section 2 is devoted to the reformulation of the Neumann boundary condition, i.e., function $G$ is introduced. In Section 3, we show that if a monotone sequence of domains is considered, then solutions of the Neumann problem in respective domains converge to the solution of a naturally defined Neumann boundary problem on the limit domain, where a proper space of test and trial functions is given. Also, the stability issue for the Neumann problem is discussed. Estimates concerning the distance between solutions on $\Omega_{\text {low }}, \Omega$ and $\Omega_{\text {up }}$ are presented in Section 4. Section 5 deals with the stability of the domain which is the limit of a nonmonotone sequence of domains. Further comments on Example 1.1 together with some conclusions constitute Section 6.

\section{The Neumann Problem defined on a SET of DOMains}

In this section, we formulate the Neumann problem in a way transferring a boundary integral into a domain integral. First we introduce an equality and then we will show its connection to the standard Neumann problem with a nonhomogeneous boundary condition. As stated in the Introduction, we focus on plane problems.

Throughout the paper, we assume any domain $\Omega$ (or $\Omega_{n}$ ), i.e., an open connected set, as well as its closure $\bar{\Omega}\left(\right.$ or $\bar{\Omega}_{n}$ ) embedded into a fixed bounded domain. Without loss of generality, we can suppose $B$ is such a superdomain. If not stated otherwise, the domains we deal with have Lipschitz boundary. We also suppose that the domain and its closure have identical boundaries, i.e., $\partial \Omega=\partial \bar{\Omega}$. Exceptions (domains with cracks) will be noted.

Let us start with some notation. The symbol $H^{k}(\Omega), k=1,2$, stands for the standard Sobolev space of square integrable functions, the generalized partial derivatives up to the order $k$ of which are square integrable on $\Omega$. The norm and the $k$ th seminorm in $H^{k}(\Omega)$ is denoted by $\|\cdot\|_{k, \Omega}$ and $|\cdot|_{k, \Omega}$, respectively. In the space $\left[L^{2}(\Omega)\right]^{m}, m=1,2$, of $m$-tuples of square integrable functions, the norm will be indicated by $\|\cdot\|_{0, \Omega}$ regardless of $m$. The subspace of all functions from $H^{1}(\Omega)$ with traces vanishing on $\partial \Omega$ is labeled by $H_{0}^{1}(\Omega)$. We will also make use of the factor space $H^{1}(\Omega) / P$, the element of which is an affine set constructed as a function from $H^{1}(\Omega)$ and all constants on $\Omega$. $H^{1}(\Omega) / P$, with the scalar product 
$(\cdot, \cdot)_{1, \Omega}$ inducing the Sobolev seminorm $|\cdot|_{1, \Omega}$, is a Hilbert space and, moreover, $|\cdot|_{1, \Omega}$ becomes its norm. $C^{1}(\bar{\Omega})$ and $C^{\infty}(\Omega)$ will denote functions continuous on $\bar{\Omega}$ up to the first derivative, and functions infinitely smooth on $\Omega$, respectively. The space of all measurable functions bounded in $\Omega$ and its norm will be symbolized by $L^{\infty}(\Omega)$ and $\|\cdot\|_{\infty, \Omega}$, respectively.

To define the problem we will study, we assume a second order elliptic operator the coefficients $a_{i j}$ of which form a $2 \times 2$ symmetric matrix $A$. We suppose $a_{i j} \in$ $L^{\infty}(B)$ and

$$
\sum_{i, j=1}^{2} a_{i j}(x) \xi_{i} \xi_{j} \geq c_{A}|\xi|_{\mathbb{R}^{2}}^{2},
$$

for each $\xi \in \mathbb{R}^{2}$ and a.a. $x \in B, c_{A}>0$ is a constant independent of $x$ and $\xi$. We also consider a function $b \in L^{\infty}(B)$. Let us introduce the following continuous and symmetric bilinear forms:

$$
\begin{aligned}
& a_{\Omega}^{0}(u, v) \stackrel{\text { def }}{=} \int_{\Omega} A \nabla u \cdot \nabla v \mathrm{~d} x, \quad u, v \in H^{1}(\Omega) ; \\
& a_{\Omega}(u, v) \stackrel{\text { def }}{=} a_{\Omega}^{0}(u, v)+\int_{\Omega} b u v \mathrm{~d} x, \quad u, v \in H^{1}(\Omega) .
\end{aligned}
$$

It holds that $c_{A}|u|_{1, \Omega}^{2} \leq a_{\Omega}^{0}(u, u)$ independently of $u$ and $\Omega$. We assume $b$ guaranteeing that a constant $c_{A b}>0$ independent of $u$ and $\Omega$ exists such that

$$
c_{A b}\|u\|_{1, \Omega}^{2} \leq a_{\Omega}(u, u), \quad u \in H^{1}(\Omega) .
$$

Let us have a function $G \in H_{0}^{1}(B) \cap H^{2}(B)$ and a Lipschitz domain $\Omega$. We define the conjugate gradient of $G$ (two dimensional rotation) by

$$
\nabla^{\star} G \stackrel{\text { def }}{=} \operatorname{curl} G \stackrel{\text { def }}{=}\left(\partial G / \partial x_{2},-\partial G / \partial x_{1}\right) .
$$

Applying the Green theorem and the orthogonality of the unit outward normal $\nu=\left(\nu_{1}, \nu_{2}\right)$ to the unit tangential vector $t=\left(-\nu_{2}, \nu_{1}\right)$, we infer

$$
\begin{aligned}
\int_{\Omega} \nabla^{\star} G \cdot \nabla v \mathrm{~d} x & =\int_{\partial \Omega} \nabla^{\star} G \cdot \nu v \mathrm{~d} s-\int_{\Omega}\left(\frac{\partial^{2} G}{\partial x_{2} \partial x_{1}}-\frac{\partial^{2} G}{\partial x_{1} \partial x_{2}}\right) v \mathrm{~d} x \\
& =\int_{\partial \Omega} \nabla G \cdot t v \mathrm{~d} s, \quad v \in H^{1}(\Omega),
\end{aligned}
$$

because the terms with second mixed derivatives in the sense of distributions cancel each other. It holds that $\left\|\nabla^{\star} G\right\|_{0, \Omega}=\|\nabla G\|_{0, \Omega}=|G|_{1, \Omega}$.

The following definition will help us to avoid a boundary integral in the Neumann problem with a nonhomogeneous boundary condition.

Let $G \in H_{0}^{1}(B)$ be fixed. We define the linear continuous functional $\int_{\partial \Omega} v \mathrm{~d} G$ operating on the space $H^{1}(\Omega)$ :

$$
\int_{\partial \Omega} v \mathrm{~d} G \stackrel{\text { def }}{=} \int_{\Omega} \nabla^{\star} G \cdot \nabla v \mathrm{~d} x, \quad v \in H^{1}(\Omega) .
$$

Let us notice that $\int_{\partial \Omega} v \mathrm{~d} G=0$ if $v \in H_{0}^{1}(\Omega)$. It is a consequence of (2.4).

The Neumann boundary value problem reads (see (2.5)): Find $u \in H^{1}(\Omega)$ such that

$$
a_{\Omega}(u, v)=\int_{\partial \Omega} v \mathrm{~d} G \quad \forall v \in H^{1}(\Omega) .
$$


Remark 2.1. If $G$ is continuously differentiable on $\bar{\Omega}$, we can define $g=\partial G / \partial s$ a.e. on $\partial \Omega$. Then $\nabla^{\star} G \cdot \nu=\nabla G \cdot t=\partial G / \partial s=g$ on the boundary, i.e.,

$$
\int_{\Omega} \nabla^{\star} G \cdot \nabla v \mathrm{~d} x=\int_{\partial \Omega} v g \mathrm{~d} s \quad \forall v \in H^{1}(\Omega) .
$$

The solution of problem (2.6) fulfills, in the weak sense, the equation $-\operatorname{div}(A \nabla u)$ $+b u=0$ with the boundary condition $\partial u / \partial \nu_{A}=g$, where $\partial u / \partial \nu_{A}$ stands for the conormal derivative.

Problem (2.6) can be considered rather strange because $\int_{\partial \Omega} \mathrm{d} G=0$ indicates that $a_{\Omega}^{0}$ would fit (2.6) better than $a_{\Omega}$. We chose (2.6) with demonstrative purposes in mind though it would make the analysis simpler if $b$ and $\nabla^{\star} G$ were avoided.

The latter happens if $\int_{\partial \Omega} g \mathrm{~d} s \neq 0$ is to be treated. Then we need two functions $G_{1}, G_{2} \in H_{0}^{1}(B)$. We define $\bar{G}=\left(G_{1}, G_{2}\right)$ and

$$
\int_{\partial \Omega} v \mathrm{~d} \bar{G} \stackrel{\text { def }}{=} \int_{\Omega} \bar{G} \cdot \nabla v \mathrm{~d} x+\int_{\Omega}\left(\frac{\partial G_{1}}{\partial x_{1}}+\frac{\partial G_{2}}{\partial x_{2}}\right) v \mathrm{~d} x, \quad v \in H^{1}(\Omega) .
$$

An analogy to Remark 2.1 is $\bar{G} \cdot \nu=g$ on $\partial \Omega$.

Let us point out that $G$ or $\bar{G}$ are viewed as primal quantities defining the problem, and $g$ is only a derived quantity which, of course, can be beneficial for an insight into the modeled problem. Functions $G, \bar{G}$ should reflect the physical background of the problem.

Example 2.1. Let us construct $\bar{G}$ for problem (1.1) in Example 1.2. For $\partial \Omega$ is the unit radius circle, it is easy to get the unit normal $\nu=\left(\nu_{1}, \nu_{2}\right)$

$$
\nu_{1}(x)=\frac{x_{1}}{r}, \quad \nu_{2}(x)=\frac{x_{2}}{r}, \quad r=\sqrt{x_{1}^{2}+x_{2}^{2}}, \quad x \in \partial \Omega .
$$

As $g=\bar{G} \cdot \nu$, we directly check

$$
G_{1}(x)=c x_{1} r^{j}, G_{2}(x)=c x_{2} r^{j} \Rightarrow g=c r^{j+1}, \quad j=\ldots,-1,0,1, \ldots,
$$

where $c$ is a constant.

Functions $\bar{G}, g$ are well defined in $\mathbb{R}^{2}$ except for the origin, where a singularity might be. We can get rid of the singularity by multiplying $\bar{G}$ by a smooth function $\chi, 0 \leq \chi \leq 1$, such that $\chi=0$ in a neighborhood of the origin and $\chi=1$ outside a circle with the center at the origin and radius, say $1 / 2$.

If $c=1$ in (2.8), then any choice results in $g=1$ on $\partial \Omega$. To pick up a specific function, we realize the fact that the greater $r$, the lower heat flux because the total amount of heat remains constant (see the Introduction). On that basis, $j=-2$ seems to be a realistic choice in (2.8).

The particular choice of $\bar{G}, G$ has no impact on convergence results achieved in Section 3. However, it determines some values in error estimates (Section 4) and this is the reason why physical background is to be taken into account.

Let us point out two facts. First, constructing $\bar{G}$, we can limit ourselves to the vicinity of $\partial \Omega$ because the fuzziness will reside right there. That is why $\chi \bar{G}$ does not cause any harm as $\chi=1$ there. Second, we can leave toothy, nonsmooth boundaries $\partial \Omega_{n}$ out of consideration. If the boundary condition has the form (2.5) or (2.7) and if $\Omega$ is stable (see the Introduction and Section 3), then $\Omega_{n} \rightarrow \Omega$ implies the convergence of the respective solutions of the BVP regardless of the smoothness of $\partial \Omega_{n}$. It explains why the naive approximation of $(1.5)$ is harmless $(G=0)$. 
Convergence details will be given in the next section. Thus, in the course of setting $G$ or $\bar{G}$, we can pay attention to smooth boundaries only.

Remark 2.2. A different approach to Example 1.2-2.1 would be to split $u$ into two parts $u=u_{0}+u_{1}$, where $u_{1}$ is a function chosen in such a way that $\int_{\partial \Omega} \partial u_{1} / \partial \nu \mathrm{d} s=$ $\int_{\partial \Omega} g \mathrm{~d} s$. As a consequence, (1.1) is transformed into a new equation for unknown $u_{0}$. It holds $\int_{\partial \Omega} \partial u_{0} / \partial \nu \mathrm{d} s=0$. Then $G$ is found for this BVP. Choosing $u_{1}=\chi \ln r$, we get $\partial u_{1} / \partial \nu=1 / r=1$ on $\partial \Omega$ (cf. Example 2.1), i.e., we can set $G$ equal to 0 or any constant.

Remark 2.3. We can also add a volume load to the problem, i.e., to seek $u \in H^{1}(\Omega)$

$$
a_{\Omega}(u, v)=\int_{\Omega} f v \mathrm{~d} x+\int_{\partial \Omega} v \mathrm{~d} \bar{G} \quad \forall v \in H^{1}(\Omega),
$$

where $f \in L^{2}(\Omega)$. If $a_{\Omega}^{0}$ is considered in (2.9), then the compatibility condition $\int_{\Omega} f \mathrm{~d} x+\int_{\partial \Omega} v \mathrm{~d} \bar{G}=0$ must be assumed. We can meet difficulties in keeping the condition if $\Omega$ is approximated by $\Omega_{n}$. If, moreover, $G$ stays instead of $\bar{G}$ in (2.9), then $\int_{\partial \Omega} 1 \mathrm{~d} G=0$. In this case, the compactness in $\Omega$ of the support of $f$ is supposed too. The latter is not necessary for the existence of a solution to (2.9) with $G$ but it simplifies arguments when a sequence of domains approaching $\Omega$ is considered (see Section 3).

In our analysis, we will only use (2.6) because (2.9) brings nothing but longer formulae as the term $\int_{\Omega} f v \mathrm{~d} x$ can be treated in a similar way as the right-hand side of (2.6) (see Sections 3 and 4).

Lemma 2.1. For any $G \in H_{0}^{1}(B)$, problem (2.6) has a unique solution $u \in H^{1}(\Omega)$.

Proof. The bilinear form $a_{\Omega}$ is continuous and $H_{1}(\Omega)$-elliptic (see $(2.3)$ ). The righthand side of (2.6) is a continuous linear functional on $H^{1}(\Omega)$ as follows from (2.5). The existence and uniqueness is due to the Lax-Milgram lemma.

Remark 2.4. If $a_{\Omega}^{0}$ is considered in (2.6), then the solution $u$ is unique in $H^{1}(\Omega) / P$. Indeed, the right-hand side of (2.6) fulfills the compatibility condition by (2.5). It equals zero if $v$ is a constant. The bilinear form $a_{\Omega}^{0}$ is continuous and $H^{1}(\Omega) / P$-elliptic. Again, the Lax-Milgram lemma finishes the proof.

Introducing the Neumann problem in the form (2.6), we have made the Neumann boundary condition easily definable on a family of subdomains of $B$.

Formulation (2.6) is advantageous for theoretical purposes. It is clumsy, however, to use (2.5) in practical computation since it would mean computing $\nabla G$ and integrating over the whole domain $\Omega$. That is why we will show some relationship between (2.5) and the Stieltjes integral.

To make use of the classical definition of the Stieltjes integral, we suppose for brevity that $\Omega$ is simply connected and we introduce the arc mapping $\beta:[0, l] \rightarrow \partial \Omega$, $\beta(0)=\beta(l), l>0$ is the length of $\partial \Omega$. Then we define the Stieltjes integral of $v$ along $\partial \Omega$ with respect to $\mathrm{d}_{S} G$ as

$$
\int_{\partial \Omega} v \mathrm{~d}_{S} G \stackrel{\text { def }}{=} \int_{0}^{l} v \circ \beta \mathrm{d}(G \circ \beta),
$$

where the integral on the right-hand side is the usual Stieltjes integral on $[0, l]$.

Lemma 2.2. Let $\Omega$ be a simply connected domain and let $G_{\beta}=G \circ \beta$ be absolutely continuous on the segment $[0, l], g_{\beta}=\mathrm{d} G_{\beta} / \mathrm{d} t$, and $g=g_{\beta} \circ \beta^{-1}: \partial \Omega \rightarrow \mathbb{R}^{1}$ 
belong to $L^{2}(\partial \Omega)$. Assume that $\left\{\varphi_{n}\right\}_{n=1}^{\infty}, \varphi_{n} \in C^{1}(\bar{\Omega})$, is a sequence converging to a function $v$ in $H^{1}(\Omega)$. Then $s_{n}=\int_{\partial \Omega} \varphi_{n} \mathrm{~d}_{S} G$ exists in the classical Stieltjes sense and $\lim _{n \rightarrow \infty} s_{n}=\int_{\partial \Omega} v \mathrm{~d} G$, where the value on the right-hand side is given by $(2.5)$.

Proof. By virtue of the Lipschitz boundary $\partial \Omega$, the existence of the sequence $\left\{\varphi_{n}\right\}_{n=1}^{\infty}$ is guaranteed due to the density of $C^{\infty}(\bar{\Omega})$ in $H^{1}(\Omega)$.

We get

$$
s_{n}=\int_{\partial \Omega} \varphi_{n} \mathrm{~d}_{S} G=\int_{0}^{l}\left(\varphi_{n} \circ \beta\right) g_{\beta} \mathrm{d} t=\int_{\partial \Omega} \varphi_{n} g \mathrm{~d} s .
$$

We find that $\lim _{n \rightarrow \infty} s_{n}=s_{0} \in \mathbb{R}^{1}$ because, due to the trace theorem,

$$
\left|s_{n}-s_{m}\right|=\left|\int_{\partial \Omega}\left(\varphi_{n}-\varphi_{m}\right) g \mathrm{~d} s\right| \leq\left\|\varphi_{n}-\varphi_{m}\right\|_{0, \partial \Omega}\|g\|_{0, \partial \Omega} \rightarrow 0
$$

if $n$ and $m$ tend to infinity.

It remains to prove that we can also arrive at $s_{0}$ via (2.5).

The function $G$ possesses the tangential derivative $\partial G / \partial s=g$ almost everywhere on $\partial \Omega$. By (2.4) we deduce

$$
\int_{\Omega} \nabla^{\star} G \cdot \nabla \varphi_{n} \mathrm{~d} x=\int_{\partial \Omega} \varphi_{n} g \mathrm{~d} s=\int_{\partial \Omega} \varphi_{n} \mathrm{~d}{ }_{S} G=s_{n} .
$$

Applying the convergence $\varphi_{n} \rightarrow v$ in $H^{1}(\Omega)$ and $s_{n} \rightarrow s_{0}$ in $\mathbb{R}^{1}$, we finish the proof by the equality

$$
\int_{\Omega} \nabla^{\star} G \cdot \nabla v \mathrm{~d} x=s_{0}
$$

Remark 2.5. Lemma 2.2 allows us to define $\int_{\partial \Omega} v \mathrm{~d}_{S} G$ for $v \in H^{1}(\Omega)$ as the limit of values $s_{n}$. If $\Omega$ is Lipschitz but not simply connected, then $\int_{\partial \Omega} v \mathrm{~d}_{S} G$ can be defined as a finite sum of Stieltjes integrals over all maximal connected components of $\partial \Omega$.

Remark 2.6. The above definitions and lemmas can be generalized even to nonLipschitzian domains. An example is a domain $\Omega$ with a cut used to model a crack. Then $G, G_{1}, G_{2} \notin H^{1}(B)$, as they have discontinuity along the crack, but $G, G_{1}, G_{2} \in H^{1}(\Omega)$. The Neumann boundary condition is defined along both sides of the crack. Calculating the Stieltjes integral, we have to follow one side of the crack to its tip and go back integrating the values on the other side.

We will consider a sequence of Neumann problems dependent on a domain. Our goal is to prove the convergence of solutions if domains converge to a limit $\Omega$. Since we do not suppose either smooth boundaries of the domains or uniform cone property, we can hardly apply the material derivative approach widely used in optimal shape design (see [HN], [HCK] $)$.

\section{Convergence Analysis}

Let us suppose that we have a sequence $\left\{\Omega_{n}\right\}_{n=1}^{\infty}$ converging to a domain $\Omega$ in the set sense, i.e., $x \in \Omega$ implies $\exists n_{x} \forall n \geq n_{x} x \in \Omega_{n}$, and $\exists y, n_{y} \forall n \geq n_{y} y \in \Omega_{n}$ implies $y \in \bar{\Omega}$. We assume $\partial \bar{\Omega}=\partial \Omega$. 
We confine ourselves to monotone sequences of domains because, as we will show later, it is sufficient to analyze the stability of $\Omega$ with respect to them. They correspond to those studied in [B1], [B2].

First we prove some convergence properties for solutions of the Neumann boundary problem defined on a sequence of monotonically expanding or shrinking domains. Then we set sufficient conditions guaranteeing that the limit functions coincide with the solution of a naturally defined problem on the limit domain (see the Introduction for the stability of a domain).

We consider almost the same equation as in (2.6):

$$
u \in H \quad a_{\Omega}(u, v)=\int_{\Omega} \nabla^{\star} G \cdot \nabla v \mathrm{~d} x \quad \forall v \in H,
$$

except for the space $H$ which will be defined later.

Let us have a sequence of subdomains $\Omega_{n}$ such that $\Omega_{n} \nearrow \Omega, n=1,2, \ldots$, i.e., $\bar{\Omega}_{n} \subset \Omega_{n+1} \subset \bar{\Omega}_{n+1} \subset \Omega$ and $\bigcup_{n=1}^{\infty} \Omega_{n}=\Omega$. We assume each $\Omega_{n}$ has the Lipschitz boundary but no such assumption is put on $\Omega$. Following the proof of $[\mathrm{B} 2$, Theorem 9.1], we define the sets $\Phi_{1}=\Omega_{1}, \Phi_{n+1}=\Omega_{n+1} \backslash \bar{\Omega}_{n}, \Psi_{n}=\bigcup_{k=n+1}^{\infty} \Phi_{k}$.

Through the sets

$$
\hat{H}_{n}=\left\{v \in L^{2}(\Omega):\left.v\right|_{\Omega_{n}} \in H^{1}\left(\Omega_{n}\right),\left.v\right|_{\Phi_{k}} \in H^{1}\left(\Phi_{k}\right), k=n+1, \ldots\right\},
$$

$n=1,2, \ldots$, and the scalar products

$$
[u, v]_{n}=\sum_{k=1}^{\infty} \int_{\Phi_{k}}(\nabla u \cdot \nabla v+u v) \mathrm{d} x, \quad n=1,2, \ldots,
$$

inducing the norms $\|\cdot\|_{n}$, we define the spaces

$$
H_{n}=\left\{v \in \hat{H}_{n}:\|v\|_{n}<\infty\right\}, \quad n=1,2, \ldots .
$$

One can see that $H_{n}$ is a Hilbert space for any $n$ and that $H_{n+1} \subset H_{n}$.

As in the proof of [B2 Theorem 9.1], it can be shown $H^{\uparrow} \stackrel{\text { def }}{=} \bigcap_{n=1}^{\infty} H_{n}$ is equivalent to $H^{1}(\Omega)$. Though B2 uses a sequence of domains with a smooth boundary and a factor space norm, the proof is applicable to our case too. Moreover, the subset of functions infinitely smooth in $\Omega$ is dense in $H^{\uparrow}$ (see [M, Theorem 1.1.5/1, 1.1.5/2]).

Lemma 3.1. Let $\Omega_{n} \nearrow \Omega$ and let $u_{n} \in H^{1}\left(\Omega_{n}\right)$ solve the equation

$$
a_{\Omega_{n}}\left(u_{n}, v\right)=\int_{\partial \Omega_{n}} v \mathrm{~d} G_{n} \quad \forall v \in H^{1}\left(\Omega_{n}\right),
$$

where $G_{n}=\left.G\right|_{\Omega_{n}}, n=1,2, \ldots, G \in H_{0}^{1}(B)$. If $\widetilde{u}_{n}$ stands for a function from $H_{n}$ equal to $u_{n}$ on $\Omega_{n}$ and to zero on $\Psi_{n}$, then $\widetilde{u}_{n} \rightarrow u_{G}$ (weakly) in any space $H_{k}$, $k=1,2, \ldots, u_{G} \in H^{\uparrow}$ and $u_{G}$ solves equation (3.1) where $H=H^{\uparrow}$.

Proof. For the sake of brevity, we will write $u$ instead of $u_{G}$ in the proof.

We have

$$
c_{A b}\left\|u_{n}\right\|_{1, \Omega_{n}}^{2} \leq a_{\Omega_{n}}\left(u_{n}, u_{n}\right) \leq\left|G_{n}\right|_{1, \Omega_{n}}\left\|u_{n}\right\|_{1, \Omega_{n}}
$$

which further implies

$$
c_{A b}\left\|u_{n}\right\|_{1, \Omega_{n}} \leq|G|_{1, B}=C,
$$

where $C$ is a positive constant independent of $n$. Passing to the sequence $\left\{\widetilde{u}_{n}\right\}_{n=1}^{\infty}$, we see that it is also bounded in any fixed space $H_{k}$ if $n \geq k$ is considered (otherwise $\widetilde{u}_{n} \notin H_{k}$ in general). 
This means that a weak limit $u^{k} \in H_{k}$ exists for a subsequence $\left\{\widetilde{u}_{n_{i}}\right\}_{i=1}^{\infty}$ of $\left\{\widetilde{u}_{n}\right\}_{n=1}^{\infty}$. We can see, however, that also $u^{k} \in H_{k+j}, j=1,2, \ldots$

Indeed, setting $j=1$, taking $v \in H_{k+j-1}$ and focusing on $\partial \Omega_{k+j-1}=\partial \Omega_{k+j-1} \cap$ $\partial \Phi_{k+j}$, we can define $u_{k+j}^{1}, v^{1}$ as the trace of $\left.u^{k}\right|_{\Omega_{k+j-1}},\left.v\right|_{\Omega_{k+j-1}}$ on $\partial \Omega_{k+j-1}$ and $u_{k+j}^{2}, v^{2}$ as the trace of $\left.u^{k}\right|_{\Phi_{k+j}},\left.v\right|_{\Phi_{k+j}}$ on $\partial \Omega_{k+j-1}$, respectively. Defining the linear continuous functional on $H_{k+j-1}$

$$
F(v)=\int_{\partial \Omega_{k+j-1}}\left(u_{k+j}^{1}-u_{k+j}^{2}\right)\left(v^{1}-v^{2}\right) \mathrm{d} s,
$$

we have $F\left(\widetilde{u}_{n}\right)=0$ for $n \geq k+j$ as $\widetilde{u}_{n} \in H_{n} \subset H_{k+j-1}, n \geq k+j$, has no jumps on $\partial \Omega_{k+j-1}$. Thus

$$
0=\lim _{i \rightarrow \infty} F\left(\widetilde{u}_{n_{i}}\right)=F\left(u^{k}\right)=\left\|u_{k+j}^{1}-u_{k+j}^{2}\right\|_{0, \Gamma_{k+j}}^{2} .
$$

Then we can add 1 to $j$ and repeat the above argument.

By this we deduce that the subsequence $\left\{\widetilde{u}_{n_{i}}\right\}_{i=1}^{\infty}$ converges to a function $u \in H^{\uparrow}$. The convergence is weak in $H_{k}$ for any fixed $k$.

We need to prove that $u$ solves (3.1) with $H=H^{\uparrow}=H^{1}(\Omega)$.

To this end we employ the technique which has proven itself useful in optimal shape design, cf. $[\mathrm{HN}]$.

Let us choose an arbitrary $v \in H^{\uparrow}$ and define a subdomain $\Omega^{m} \subset \Omega$,

$$
\Omega^{m}=\{x \in \Omega: \operatorname{dist}(x, \partial \Omega)>1 / m\} .
$$

If $n_{i}$ is sufficiently large, we have

$$
\begin{aligned}
I_{1}(i) & \equiv a_{\Omega_{n_{i}}}\left(u_{n_{i}}, v\right) \\
& =\int_{\Omega_{n_{i}} \backslash \Omega^{m}}\left(A \nabla u_{n_{i}} \cdot \nabla v+b u_{n_{i}} v\right) \mathrm{d} x+a_{\Omega^{m}}\left(u_{n_{i}}, v\right) \\
& \equiv I_{11}(i, m)+a_{\Omega^{m}}\left(u_{n_{i}}, v\right) .
\end{aligned}
$$

By the boundedness of $A, b$ and $u_{n_{i}}$, we can estimate

$$
\left|I_{11}(i, m)\right| \leq C_{1}\left\|u_{n_{i}}\right\|_{1, \Omega_{n_{i}}}\|v\|_{1, \Omega \backslash \Omega^{m}} \leq C_{2}\|v\|_{1, \Omega \backslash \Omega^{m}} \equiv C_{2} I(m),
$$

where $C_{2}>0$ does not depend on $i, m$.

For $v$ fixed, $a_{\Omega^{m}}\left(u_{n_{i}}, v\right)$ is a linear continuous functional on $H_{k}, k$ arbitrary. By the weak convergence of $u_{n_{i}}$ and $H^{\uparrow} \subset H_{k}$,

$$
\lim _{i \rightarrow \infty} a_{\Omega^{m}}\left(u_{n_{i}}, v\right)=a_{\Omega^{m}}(u, v) .
$$

On the basis of (3.5) and (3.6),

$$
-C_{2} I(m)+a_{\Omega^{m}}(u, v) \leq \liminf _{i \rightarrow \infty} I_{1}(i) \leq \limsup _{i \rightarrow \infty} I_{1}(i) \leq C_{2} I(m)+a_{\Omega^{m}}(u, v) .
$$

The parameter $m$ can be arbitrarily large causing $I(m) \rightarrow 0$ and, consequently,

$$
\lim _{i \rightarrow \infty} I_{1}(i)=a_{\Omega}(u, v) .
$$

We also have

$$
\lim _{i \rightarrow \infty} \int_{\Omega_{n_{i}}} \nabla^{\star} G \cdot \nabla v \mathrm{~d} x=\int_{\Omega} \nabla^{\star} G \cdot \nabla v \mathrm{~d} x .
$$

Combining (3.2), (3.7), and (3.8), we get (3.1) for a fixed but arbitrary function $v \in H^{\uparrow}$, i.e., $u$ solves problem (3.1). 
We also deduce that the limit function of any weakly convergent subsequence of $\left\{\widetilde{u}_{n}\right\}_{n=1}^{\infty}$ is a solution to (3.1). The solution is unique in $H^{1}(\Omega)$, thus the whole sequence converges weakly to $u$.

We can even prove convergence in a stronger sense.

Lemma 3.2. Under the assumptions of Lemma 3.1,

$$
\lim _{n \rightarrow \infty} a_{\Omega_{n}}\left(u_{G}-u_{n}, u_{G}-u_{n}\right)=0
$$

Proof. Again, we abbreviate $u=u_{G}$ in the proof. Using (3.2) and (3.1), we estimate

$$
\begin{aligned}
I(n) & \equiv a_{\Omega_{n}}\left(u-u_{n}, u-u_{n}\right) \leq a_{\Omega}(u, u)-2 a_{\Omega_{n}}\left(u, u_{n}\right)+a_{\Omega_{n}}\left(u_{n}, u_{n}\right) \\
& =\int_{\Omega} \nabla^{\star} G \cdot \nabla u \mathrm{~d} x-2 \int_{\Omega_{n}} \nabla^{\star} G \cdot \nabla u \mathrm{~d} x+\int_{\Omega_{n}} \nabla^{\star} G \cdot \nabla u_{n} \mathrm{~d} x \\
& \equiv I_{1}-2 I_{2}(n)+I_{3}(n) .
\end{aligned}
$$

As in the proof of Lemma 3.1 (cf. (3.8)), we have

$$
\lim _{n \rightarrow \infty} I_{2}(n)=\int_{\Omega} \nabla^{\star} G \cdot \nabla u \mathrm{~d} x .
$$

Integrating separately over $\Omega^{m}$ and $\Omega_{n} \backslash \Omega^{m}$ (see (3.4) for $\Omega^{m}$ ), we get

$$
\limsup _{n \rightarrow \infty} I_{3}(n) \leq C|G|_{1, \Omega \backslash \Omega^{m}}+\int_{\Omega^{m}} \nabla^{\star} G \cdot \nabla u \mathrm{~d} x,
$$

where $C>0$ does not depend on $m$. To infer (3.11) we made use of the weak convergence and boundedness of $\left\{u_{n}\right\}_{n=1}^{\infty}$.

Taking into account (3.9)-(3.11), we can estimate

$$
\begin{aligned}
0 \leq \limsup _{n \rightarrow \infty} I(n) & \leq-\int_{\Omega} \nabla^{\star} G \cdot \nabla u \mathrm{~d} x+C|G|_{1, \Omega \backslash \Omega^{m}}+\int_{\Omega^{m}} \nabla^{\star} G \cdot \nabla u \mathrm{~d} x \\
& =-\int_{\Omega \backslash \Omega^{m}} \nabla^{\star} G \cdot \nabla u \mathrm{~d} x+C|G|_{1, \Omega \backslash \Omega^{m}} .
\end{aligned}
$$

Since the magnitude of $m$ is arbitrary, $\lim _{n \rightarrow \infty} I(n)=0$.

Remark 3.1. Lemmas 3.1 and 3.2 remain valid even if $\Omega$ is a domain with a crack approached by an increasing sequence of subdomains $\Omega_{n}$. If $\Omega_{n} \nearrow \Omega$, then we do not need to assume $\partial \Omega=\partial \bar{\Omega}$. The Neumann problem is stable from inside for any $\Omega$.

Remark 3.2. To reformulate Lemmas 3.1 and 3.2 if $a_{\Omega}^{0}$ is substituted for $a_{\Omega}$, we have to define $H_{n}$ as factor spaces with respect to the space of constants, omit the nondifferentiated term in the definition of $[u, v]_{n}$, and use the seminorm $\left|u_{n}\right|_{1, \Omega_{n}}$ instead of $\left\|u_{n}\right\|_{1, \Omega_{n}}$ in (3.3). The proofs remain basically unchanged.

We will focus on a sequence $\Omega_{n} \searrow \Omega$ now, i.e., $\bar{\Omega} \subset \Omega_{n+1} \subset \bar{\Omega}_{n+1} \subset \Omega_{n}$ and $\bigcap_{n=1}^{\infty} \Omega_{n}=\bar{\Omega}$.

Unlike the previous case, we simply define $H_{n}=H^{1}\left(\Omega_{n}\right)$. The Hilbert space $H^{\downarrow}$ is defined as the $\|\cdot\|_{1, \Omega}$-closure of all functions continuous together with their first derivatives on a neighborhood of $\bar{\Omega}$. In general, $H^{\downarrow} \subset H^{\uparrow}=H^{1}(\Omega)$. 
Lemma 3.3. Let $\Omega_{n} \searrow \Omega$ and $u_{n} \in H_{n}$ solve equation (3.2). Then

$$
\lim _{n \rightarrow \infty} a_{\Omega}\left(u^{G}-u_{n}, u^{G}-u_{n}\right)=0,
$$

where $u^{G} \in H=H^{\downarrow}$ solves (3.1).

Proof. As before, we will simply write $u$ instead of $u^{G}$ hereafter. By an argument similar to that used to infer (3.3),

$$
c_{A b}\left\|u_{n}\right\|_{1, \Omega} \leq c_{A b}\left\|u_{n}\right\|_{1, \Omega_{n}} \leq C,
$$

where $C>0$ is independent of $n$. Again, a sequence $\left\{\left.u_{n_{i}}\right|_{\Omega}\right\}_{i=1}^{\infty}$ converging weakly to a function $u \in H^{\downarrow}$ exists. Indeed, any $u_{n}$ falls into the $H^{1}\left(\Omega_{n}\right)$-closure of smooth functions on $\Omega_{n}$, i.e., $\left.u_{n}\right|_{\Omega} \in H^{\downarrow}$ because $\bar{\Omega} \subset \Omega_{n}$.

To prove that $u$ solves (3.1), we choose an arbitrary function $\varphi$ such that it is continuous together with its first partial derivatives on a domain $\Omega_{\delta} \supset \bar{\Omega}$, i.e., $\left.\varphi\right|_{\Omega} \in H^{\downarrow}$.

If $i$ is sufficiently large, then we have $\bar{\Omega}_{n_{i}} \subset \Omega_{\delta}$ which implies $\left.\varphi\right|_{\Omega_{n i}} \in H^{1}\left(\Omega_{n i}\right)$. We introduce

$$
I_{1}\left(n_{i}\right) \equiv a_{\Omega_{n_{i}}}\left(u_{n_{i}}, \varphi\right)=a_{\Omega_{n_{i}} \backslash \Omega}\left(u_{n_{i}}, \varphi\right)+a_{\Omega}\left(u_{n_{i}}, \varphi\right) .
$$

Following the proof of (3.7), we deduce from the weak convergence of $\left\{\left.u_{n_{i}}\right|_{\Omega}\right\}_{i=1}^{\infty}$

$$
\lim _{i \rightarrow \infty} I_{1}\left(n_{i}\right)=a_{\Omega}(u, \varphi) \text {. }
$$

We also have

$$
\lim _{i \rightarrow \infty} \int_{\Omega_{n_{i}}} \nabla^{\star} G \cdot \nabla \varphi \mathrm{d} x=\int_{\Omega} \nabla^{\star} G \cdot \nabla \varphi \mathrm{d} x
$$

which, together with (3.14), proves equality (3.1) for an arbitrary smooth test function $\varphi$. By virtue of the density argument, we conclude that $u$ is the solution of problem (3.1) with $H=H^{\downarrow}$.

Using the uniqueness argument, we infer that the whole sequence $\left\{u_{n}\right\}_{n=1}^{\infty}$ converges to $u$ weakly in $H$.

Let us focus on (3.12), i.e., on

$$
0 \leq I(n) \equiv a_{\Omega}\left(u-u_{n}, u-u_{n}\right) \leq a_{\Omega}(u, u)-2 a_{\Omega}\left(u, u_{n}\right)+I_{2}(n),
$$

where

$$
\begin{aligned}
I_{2}(n) & \equiv a_{\Omega_{n}}\left(u_{n}, u_{n}\right) \\
& =\int_{\Omega_{n} \backslash \Omega} \nabla^{\star} G \cdot \nabla u_{n} \mathrm{~d} x+\int_{\Omega} \nabla^{\star} G \cdot \nabla u_{n} \mathrm{~d} x \equiv I_{21}(n)+I_{22}(n) .
\end{aligned}
$$

We get

$$
\lim _{n \rightarrow \infty} I_{2}(n)=\int_{\Omega} \nabla^{\star} G \cdot \nabla u \mathrm{~d} x=a_{\Omega}(u, u)
$$

as a consequence of $\left|I_{21}(n)\right| \leq C a_{A b}^{-1}|G|_{1, \Omega_{n} \backslash \Omega}$ (see (3.13)) and the weak convergence $u_{n} \rightarrow u$.

Using the latter, (3.16), and passing to the limit in (3.15), we finish the proof by

$$
\lim _{n \rightarrow \infty} I(n)=0 \text {. }
$$


We have shown that $\Omega$ is stable from outside and $H^{\downarrow} \subset H^{\uparrow}$. The latter admits $H^{\downarrow} \neq H^{\uparrow}$.

If $H^{\downarrow}=H^{\uparrow}$, then $u_{G}=u^{G}$ and $\Omega$ is stable in the sense that the limit of solutions coincides with the unique solution of the Neumann problem on the limit domain $\Omega$.

If $\Omega$ is stable with respect to monotone sequences of domains, then it is also stable with respect to any (including nonmonotone) sequence $\left\{\Omega_{n}\right\}_{n=1}^{\infty}$ converging to $\Omega$ in the set sense. We postpone the proof because we will need some results contained in Section 4. The general stability issue will be treated in Section 5 .

Our next goal is to ensure the continuity of solutions of the Neumann problem with respect to a sequence of domains $\Omega_{n}$. We wish to characterize the stable domain.

We use different definitions of the space $H$ in Lemma 3.1 and Lemma 3.3, respectively, and we need to get an identical space in both cases. To guarantee that $\Omega$ is stable with respect to the Neumann problem (N-stable), it is not sufficient to have only $\partial \Omega=\partial \bar{\Omega}$ (see [M, page 14] or $[\underline{\mathrm{K}}$ for a counterexample).

Let us remind the $\sigma$ property as defined in [B2, Definition 5.3].

Definition 3.1. A domain $\Omega \subset \bar{\Omega} \subset B$ has the $\sigma$ property if for any point $X \in \partial \Omega$ there exist an open ball $B_{X} \subset \bar{B}_{X} \subset B$ with the center $X$ and a vector $0 \neq v_{X} \in \mathbb{R}^{2}$ such that ${\overline{\left(B_{X} \cap \Omega\right)}}_{t v_{X}} \subset \Omega$ for any $t \in(0,1]$, where $\left(B_{X} \cap \Omega\right)_{t v_{X}}=\left\{x \in \mathbb{R}^{2}\right.$ : $\left.x+t v_{X} \in B_{X} \cap \Omega\right\}$.

If the boundary of a domain can be locally defined by a function, then the domain has the $\sigma$ property (see [B2, Remark 3, p. 170]).

Let us recall that a domain $\Omega$ is called starshaped if a point $z \in \Omega$ exists such that any ray with origin $z$ has a unique common point with $\partial \Omega$.

The following theorem addresses the stability problem.

Theorem 3.1. Let us have sequences $\left\{u_{n_{1}}\right\}_{n_{1}=1}^{\infty}$ and $\left\{u_{n_{2}}\right\}_{n_{2}=1}^{\infty}$ of solutions of the Neumann problem on domains $\Omega_{n_{1}} \nearrow \Omega$ and $\Omega_{n_{2}} \searrow \Omega$, respectively. Let $\partial \Omega=\partial \bar{\Omega}$ and $\Omega$ be starshaped or have the $\sigma$ property. Then for any $G \in H_{0}^{1}(B), u_{G}=u^{G}$ in Lemmas 3.1-3.3.

Proof. If $\Omega$ is starshaped, then, due to [M, Theorem 1.1.6/1], the space $C^{\infty}(\bar{\Omega})$ is dense in $H^{1}(\Omega)$, i.e., $H^{\downarrow}=H^{\uparrow}$ (see also [B2, Theorem 9.3]).

According to [B2, Theorem 9.4], $\Omega$ is N-stable with respect to a $k$-harmonic operator if it belongs to the Nikodym family of domains and possesses the $\sigma$ property. The proof refers to [B2, Theorem 5.5] and is directly applicable to the operator defined through $a_{\Omega}$ from (2.2). In that case, the $\sigma$ property alone is sufficient to enforce the N-stability because the Nikodym domain assumption is no longer needed as the space $H^{\downarrow}$ is the closure in the $H^{1}$-norm (see also Remark 3.3).

Remark 3.3. As regards $a_{\Omega}^{0}$, Lemma 3.3 holds with $a_{\Omega}^{0}$ in (3.12) and the first seminorms in (3.13). The space $H^{\downarrow}$ is given as the closure in $|\cdot|_{1, \Omega}$ seminorm which becomes a norm in spaces factored with respect to constants. In general, elements of $H^{\downarrow}$ can be distributions the first partial derivatives of which are square integrable functions.

Then Theorem 3.1 is also valid under the assumption that $\partial \Omega=\partial \bar{\Omega}$, $\Omega$ has the $\sigma$ property, and $\Omega$ belongs to the Nikodym family of domains (see [B2, Theorem 9.4]). Let us recall that $\Omega$ is a Nikodym domain if any function, the first generalized derivatives of which are square integrable, is also square integrable on $\Omega$. A sufficient 
condition for being a domain of the Nikodym type is the cone property (see [B2, Remark 1, p. 200]), which is obviously satisfied if $\Omega$ is Lipschitz, for instance.

Remark 3.4. The technique used in this section to prove convergence can be directly applied to problem (2.9) with volume loads and (2.7) instead of (2.5). Functions $f$, $G_{1}$ and $G_{2}$ can be treated in a similar way as function $G$.

Remark 3.5. In our formulation, the stability of the domain with respect to the Neumann boundary value problem is the same for homogeneous and nonhomogeneous boundary conditions. It is not true for the classical formulation (cf. Example 1.2).

\section{Estimates}

In the previous section, we proved convergence of solutions of the Neumann problem. In the current one, we will estimate the difference between solutions on different but "close" domains. To make ideas more lucid, we start with a general estimate and then temporarily confine ourselves to a rather special class of domains. Besides the norm $\|\cdot\|_{1, \Omega}$ we will also use the energy norm $\|\cdot\|_{A, \Omega} \stackrel{\text { def }}{=}\left(a_{\Omega}(\cdot, \cdot)\right)^{1 / 2}$ and seminorm $|\cdot|_{A, \Omega} \stackrel{\text { def }}{=}\left(a_{\Omega}^{0}(\cdot, \cdot)\right)^{1 / 2}$. By $(2.2)-(2.3)$ both norms are equivalent.

Let domains $\Omega_{\text {low }}, \Omega_{\text {up }}$ and $\Omega, \bar{\Omega}_{\text {low }} \subset \Omega \subset \bar{\Omega} \subset \Omega_{\text {up }} \subset \bar{\Omega}_{\text {up }} \subset B \subset \mathbb{R}^{2}$, be given such that $\partial \Omega_{\text {low }}, \partial \Omega_{\text {up }}$ are Lipschitz and $\partial \Omega=\partial \bar{\Omega}$ but $\Omega$ is not necessarily N-stable. If $\Omega$ is unstable, then $H^{\downarrow} \varsubsetneqq H^{\uparrow}=H^{1}(\Omega)$, where $H^{\downarrow}$ is defined by means of a sequence $\left\{\Omega_{n}\right\}_{n=1}^{\infty}, \Omega_{n} \searrow \Omega$. Approaching $\Omega$ by a nonmonotone sequence $\Omega_{m} \rightarrow \Omega$, we could get that the respective solutions $u_{m}$ either do not converge or converge to a function $\widetilde{u} \in \widetilde{H}$ solving (3.1) with $H=\widetilde{H}$, where $\widetilde{H}$ is a space such that $H^{\downarrow} \subset \widetilde{H} \subset H^{\uparrow}$. It can be $u_{G} \neq \widetilde{u} \neq u^{G}$. Though $\Omega$ could be N-unstable, it can still be approximated by reasonable domains $\Omega_{\text {low }}$ and $\Omega_{\text {up }}$. This offers a possibility to approximate $\widetilde{u}$ by the solution of the Neumann boundary value problem on $\Omega_{\text {low }}$ or $\Omega_{\text {up }}$.

Having $\Omega_{m} \rightarrow \Omega$, we observe that $\bar{\Omega}_{\text {low }} \subset \Omega_{m} \subset \bar{\Omega}_{m} \subset \Omega_{\text {up }}$ for sufficiently large $m$. As in the previous paragraph, we can ask what the difference between $u_{m}$ and the solution on $\Omega_{\text {up }}$ (or $\Omega_{\text {low }}$ ) might be.

We denote the solution of (3.1) defined for $\Omega_{1} \stackrel{\text { def }}{=} \Omega_{\text {low }}, H=H^{1}\left(\Omega_{1}\right)$, and $\Omega_{3} \stackrel{\text { def }}{=} \Omega_{\text {up }}$, $H=H^{1}\left(\Omega_{3}\right)$ by $u_{1}$ and $u_{3}$, respectively. Let $u_{2}$ be the solution of (3.1) on a domain between $\Omega_{1}$ and $\Omega_{3}$.

We can choose between several possibilities. If $\Omega$ is N-unstable and the abovementioned space $\widetilde{H}$ is considered, then we can set $\Omega_{2}=\Omega$ and $u_{2}$ equivalent to the solution of (3.1) with $H=\widetilde{H}, H^{\downarrow} \subset \widetilde{H} \subset H^{\uparrow}$. If $\Omega$ is N-stable, then $\Omega_{2}=\Omega$ and $u_{2}$ solves (3.1) with $H=\widetilde{H}=H^{1}(\Omega)$. If $\Omega_{m}$ is in focus instead of $\Omega$, then we can put $\Omega_{2}=\Omega_{m}$ and $u_{2} \in H$ solves (3.1) with $H=H^{1}\left(\Omega_{m}\right)$.

As the estimates will depend neither on the stability assumption nor on our particular choice of $\Omega_{2}$ and $u_{2}$, any fixation of $\Omega_{2}$ and $u_{2}$ is the matter of formalism. We stick to $\Omega_{2} \stackrel{\text { def }}{=} \Omega$ and $u_{2} \in \widetilde{H}$.

We will also need $u_{12}$ and $u_{23}$, solutions to auxiliary problems on $\Omega_{12} \stackrel{\text { def }}{=} \Omega_{2} \backslash \bar{\Omega}_{1}$ and $\Omega_{23} \stackrel{\text { def }}{=} \Omega_{3} \backslash \bar{\Omega}_{2}$, respectively. We define $\Omega_{13} \stackrel{\text { def }}{=} \Omega_{3} \backslash \bar{\Omega}_{1}$. 
We seek $u_{12} \in H^{1}\left(\Omega_{12}\right), u_{23} \in H^{1}\left(\Omega_{23}\right)$ such that

$$
\begin{array}{ll}
\int_{\Omega_{12}}\left(A \nabla u_{12} \cdot \nabla v+b u_{12} v\right) \mathrm{d} x=\int_{\Omega_{12}} \nabla^{\star} G \cdot \nabla v \mathrm{~d} x & \forall v \in H^{1}\left(\Omega_{12}\right), \\
\int_{\Omega_{23}}\left(A \nabla u_{23} \cdot \nabla v+b u_{23} v\right) \mathrm{d} x=\int_{\Omega_{23}} \nabla^{\star} G \cdot \nabla v \mathrm{~d} x & \forall v \in H^{1}\left(\Omega_{23}\right) .
\end{array}
$$

Based on (4.1), the estimate

$$
\left\|u_{i}\right\|_{A, \Omega_{i}}^{2}=\int_{\Omega_{i}} \nabla^{\star} G \cdot \nabla u_{i} \mathrm{~d} x \leq|G|_{1, \Omega_{i}}\left|u_{i}\right|_{1, \Omega_{i}}, \quad i=12,23,
$$

provides us with some assessment of $u_{12}, u_{23}$ if we put an assumption on $G \in H^{1}(B)$ and apply the Schwarz inequality to (4.2).

Lemma 4.1. Assume $\nabla G \in\left[L^{\infty}\left(\Omega_{i}\right)\right]^{2}, i=12,23$. Then

$$
\left\|u_{i}\right\|_{A, \Omega_{i}} \leq \sqrt{2}\left(\operatorname{meas} \Omega_{i}\right)^{1 / 2} c_{A b}^{-1 / 2}\|\nabla G\|_{\infty, \Omega_{i}}
$$

where $\|\nabla G\|_{\infty, \Omega_{i}}=\max \left(\left\|\partial G / \partial x_{1}\right\|_{\infty, \Omega_{i}},\left\|\partial G / \partial x_{2}\right\|_{\infty, \Omega_{i}}\right)$.

Let us introduce the space $H_{12}=H^{1}\left(\Omega_{1}\right) \times H^{1}\left(\Omega_{12}\right)$ and $H_{23}=H^{1}\left(\Omega_{2}\right) \times$ $H^{1}\left(\Omega_{23}\right)$ endowed with the scalar product and the norm induced by the scalar product and the norm on respective component spaces. We can use the same symbols as for the scalar product and the norm on $H^{1}\left(\Omega_{2}\right)$ as well as $H^{1}\left(\Omega_{3}\right)$ because

$$
\operatorname{meas}\left(\Omega_{2} \backslash\left(\Omega_{1} \cup \Omega_{12}\right)\right)=0=\operatorname{meas}\left(\Omega_{3} \backslash\left(\Omega_{2} \cup \Omega_{23}\right)\right) .
$$

The following lemma shows a relationship between solutions of the Neumann problems on embedded domains. The couple $\left(u_{1}, u_{12}\right)$ belongs to $H_{12}$ and $\left(u_{2}, u_{23}\right) \in$ $H_{23}$.

Lemma 4.2. Assume $G \in H^{1}\left(\Omega_{3}\right)$. Let $P_{\Omega_{2}}: H_{12} \rightarrow \widetilde{H}$ and $P_{\Omega_{3}}: H_{23} \rightarrow H^{1}\left(\Omega_{3}\right)$ be the $(\cdot, \cdot)_{A, \Omega_{2}}$-orthogonal and the $(\cdot, \cdot)_{A, \Omega_{3}}$-orthogonal projection mapping, respectively. Then $P_{\Omega_{2}}\left(u_{1}, u_{12}\right)=u_{2}, P_{\Omega_{3}}\left(u_{2}, u_{23}\right)=u_{3}$, and

$$
\left\|u_{2}\right\|_{A, \Omega_{2}}^{2} \leq\left\|u_{1}\right\|_{A, \Omega_{1}}^{2}+\left\|u_{12}\right\|_{A, \Omega_{12}}^{2}, \quad\left\|u_{3}\right\|_{A, \Omega_{3}}^{2} \leq\left\|u_{2}\right\|_{A, \Omega_{2}}^{2}+\left\|u_{23}\right\|_{A, \Omega_{23}}^{2} .
$$

Proof. Let us notice that if $v \in \widetilde{H}$, then $\left.v\right|_{\Omega_{1}} \in H^{1}\left(\Omega_{1}\right)$ and $\left.v\right|_{\Omega_{12}} \in H^{1}\left(\Omega_{12}\right)$ as $\widetilde{H} \subset H^{1}\left(\Omega_{2}\right)$. It is easy to show $u_{2}=P_{\Omega_{2}}\left(u_{1}, u_{12}\right)$; i.e., for any $v \in \widetilde{H}$

$$
\begin{aligned}
& \left(\left(u_{1}, u_{12}\right)-u_{2}, v\right)_{A, \Omega_{2}}=a_{\Omega_{1}}\left(u_{1}, v\right)+a_{\Omega_{12}}\left(u_{12}, v\right)-a_{\Omega_{2}}\left(u_{2}, v\right) \\
& =\int_{\Omega_{1}} \nabla^{\star} G \cdot \nabla v \mathrm{~d} x+\int_{\Omega_{12}} \nabla^{\star} G \cdot \nabla v \mathrm{~d} x-\int_{\Omega_{2}} \nabla^{\star} G \cdot \nabla v \mathrm{~d} x=0 .
\end{aligned}
$$

Similarly, $u_{3}=P_{\Omega_{3}}\left(u_{2}, u_{23}\right)$ as $\left(\left(u_{2}, u_{23}\right)-u_{3}, v\right)_{A, \Omega_{3}}=0$ due to $\left.v\right|_{\Omega_{2}} \in H^{\downarrow} \subset \widetilde{H}$ if $v \in H^{1}\left(\Omega_{3}\right)$.

The norm of the projection mappings $P_{\Omega_{2}}, P_{\Omega_{3}}$ is equal to 1 , which implies inequalities (4.4). 
We can derive one simple observation from Lemma 4.2. Since $\left(u_{1}, u_{12}\right)-u_{2}$ and $u_{2}$ are orthogonal with respect to $(\cdot, \cdot)_{A, \Omega_{2}}$ and (4.4) holds, we get

$$
\begin{aligned}
\left\|u_{2}-u_{1}\right\|_{A, \Omega_{1}}^{2} & \leq\left\|\left(u_{1}, u_{12}\right)-u_{2}\right\|_{A, \Omega_{2}}^{2} \\
& =\left(\left(u_{1}, u_{12}\right),\left(u_{1}, u_{12}\right)\right)_{A, \Omega_{2}}-\left(u_{2},\left(u_{1}, u_{12}\right)-u_{2}+u_{2}\right)_{A, \Omega_{2}} \\
& =\left\|u_{1}\right\|_{A, \Omega_{1}}^{2}+\left\|u_{12}\right\|_{A, \Omega_{12}}^{2}-\left\|u_{2}\right\|_{A, \Omega_{2}}^{2} \\
& \leq\left\|u_{1}\right\|_{A, \Omega_{1}}^{2}-\left\|u_{3}\right\|_{A, \Omega_{3}}^{2}+\left\|u_{12}\right\|_{A, \Omega_{12}}^{2}+\left\|u_{23}\right\|_{A, \Omega_{23}}^{2} .
\end{aligned}
$$

Remark 4.1. Estimate (4.5) gives a hint for computation. We can approximate the unknown domain $\Omega_{2}$ from inside and outside by $\Omega_{1}$ and $\Omega_{3}$, respectively. Then we approximate $u_{1}, u_{3}$ by a numerical solution and estimate $\left\|u_{12}\right\|_{A, \Omega_{12}}^{2}+\left\|u_{23}\right\|_{A, \Omega_{23}}^{2}$ by Lemma 4.1 .

We will be interested in the value $\left\|u_{2}-u_{1}\right\|_{A, \Omega_{1}}$. We already know that, under some assumptions, $\left\|u_{12}\right\|_{A, \Omega_{12}}^{2}$ and $\left\|u_{23}\right\|_{A, \Omega_{23}}^{2}$ can be "small" quantities (see Lemma 4.1). We also feel that if $\Omega_{1}$ and $\Omega_{3}$ are not much different, then the same should hold for respective norms of $u_{1}$ and $u_{3}$. Plugging such results into (4.5), we would arrive at a desired estimate.

The previous paragraph describes our goal for what follows. First, however, we confine ourselves to a particular family of domains. To simplify the notation, we also assume that $b$ as well as all entries of the matrix $A$ are constants. A generalization to nonconstant $A$ and $b$ is straightforward and only technical.

Starshaped domains. Throughout this subsection, we deal with domains $\Omega_{1}, \Omega_{2}$, and $\Omega_{3}$ having the following properties: $\Omega_{1}$ is a domain starshaped with respect to the origin of the coordinate system,

$$
\Omega_{3}=\left\{y \in \mathbb{R}^{2}: y / \alpha \in \Omega_{1}\right\},
$$

where $\alpha>1$ is a given constant, and $\bar{\Omega}_{1} \subset \Omega_{2} \subset \bar{\Omega}_{2} \subset \Omega_{3} \subset \bar{\Omega}_{3} \subset B$. Domain $\Omega_{2}$ can be N-unstable.

As in previous paragraphs, we use subscripts 1,2,3 to tag the solution $u$ of (3.1) respective to the domains just introduced.

We define mapping $\varkappa(x)=\alpha x, y=\varkappa(x)$, which maps $\Omega_{1}$ onto $\Omega_{3}$. If a function $u$ is differentiable on $\Omega_{1}$ and $v(y)=v(\varkappa(x))=u(x)$, then elementary calculus leads to

$$
\frac{\partial v}{\partial y_{1}}=\left(\frac{\partial u}{\partial x_{1}} \frac{\partial \varkappa_{2}}{\partial x_{2}}-\frac{\partial u}{\partial x_{2}} \frac{\partial \varkappa_{2}}{\partial x_{1}}\right) / D, \quad \frac{\partial v}{\partial y_{2}}=\left(\frac{\partial u}{\partial x_{2}} \frac{\partial \varkappa_{1}}{\partial x_{1}}-\frac{\partial u}{\partial x_{1}} \frac{\partial \varkappa_{1}}{\partial x_{2}}\right) / D
$$

where $D=\partial y_{1} / \partial x_{1} \partial y_{2} / \partial x_{2}-\partial y_{2} / \partial x_{1} \partial y_{1} / \partial x_{2}$.

In our particular case,

$$
D=\alpha^{2}, \quad \partial v / \partial y_{1}=\alpha^{-1} \partial u / \partial x_{1}, \quad \partial v / \partial y_{2}=\alpha^{-1} \partial u / \partial x_{2} .
$$

As a consequence, we can infer, using the substitution theorem, that if $v \in H^{1}\left(\Omega_{3}\right)$, $v(y)=v(\varkappa(x))=u(x), u \in H^{1}\left(\Omega_{1}\right)$, then

$$
\begin{gathered}
|v|_{1, \Omega_{3}}=|u|_{1, \Omega_{1}}, \quad|v|_{A, \Omega_{3}}=|u|_{A, \Omega_{1}}, \\
\|v\|_{1, \Omega_{3}}^{2}=|u|_{1, \Omega_{1}}^{2}+\alpha^{2}|u|_{0, \Omega_{1}}, \\
\|v\|_{A, \Omega_{3}}^{2}=|u|_{A, \Omega_{1}}^{2}+\alpha^{2} b|u|_{0, \Omega_{1}}^{2} \geq\|u\|_{A, \Omega_{1}}^{2} .
\end{gathered}
$$


We can transform the solution $u_{1}$ into $u_{1 \alpha}(y)=u_{1 \alpha}(\varkappa(x))=u_{1}(x)$ and compare it to $u_{3}$ because both functions are defined on $\Omega_{3}$. To this end we define $G_{1 \alpha}(y)=$ $G_{1 \alpha}(\varkappa(x))=G_{1}(x),\left.G_{1} \equiv G\right|_{\Omega_{1}}$ and formulate a few auxiliary lemmas.

Lemma 4.3. Function $u_{1 \alpha} \in H^{1}\left(\Omega_{3}\right)$ solves the equation

$$
\int_{\Omega_{3}}\left(A \nabla u_{1 \alpha} \cdot \nabla v+\alpha^{-2} b u_{1 \alpha} v\right) \mathrm{d} y=\int_{\Omega_{3}} \nabla^{\star} G_{1 \alpha} \cdot \nabla v \mathrm{~d} y \quad \forall v \in H^{1}\left(\Omega_{3}\right) .
$$

Proof. We introduce $\widehat{v}(x)=v(\varkappa(x))$. Then (4.7) and the substitution theorem give

$$
\begin{aligned}
\int_{\Omega_{3}}( & \left.A \nabla_{y} u_{1 \alpha} \cdot \nabla_{y} v+\alpha^{-2} b u_{1 \alpha} v\right) \mathrm{d} y \\
\quad= & \int_{\Omega_{1}}\left[A\left(\alpha^{-1} \nabla_{x} u_{1}\right) \cdot\left(\alpha^{-1} \nabla_{x} \widehat{v}\right) \alpha^{2}+b u_{1} \widehat{v}\right] \mathrm{d} x \\
= & a_{\Omega_{1}}\left(u_{1}, \widehat{v}\right)=\int_{\Omega_{1}} \nabla_{x}^{\star} G_{1} \cdot \nabla_{x} \widehat{v} \alpha^{2} / \alpha^{2} \mathrm{~d} x=\int_{\Omega_{3}} \nabla_{y}^{\star} G_{1 \alpha} \cdot \nabla_{y} v \mathrm{~d} y,
\end{aligned}
$$

where subscripts $x$ and $y$ indicate the variable we use in differentiation.

Referring to the equalities

$$
\begin{aligned}
a_{\Omega_{3}}\left(u_{3}-u_{1 \alpha}, v\right) \\
=\int_{\Omega_{3}}\left[A \nabla\left(u_{3}-u_{1 \alpha}\right) \cdot \nabla v+b\left(u_{3}-u_{1 \alpha}\right) v\right] \mathrm{d} y \\
=\int_{\Omega_{3}} \nabla^{\star} G \cdot \nabla v \mathrm{~d} y \\
\quad-\int_{\Omega_{3}}\left[A \nabla u_{1 \alpha} \cdot \nabla v+\alpha^{-2} b u_{1 \alpha} v\right] \mathrm{d} y-\left(\alpha^{2}-1\right) \alpha^{-2} \int_{\Omega_{3}} b u_{1 \alpha} v \mathrm{~d} y \\
=\int_{\Omega_{3}} \nabla^{\star} G \cdot \nabla v \mathrm{~d} y-\int_{\Omega_{3}} \nabla^{\star} G_{1 \alpha} \cdot \nabla v \mathrm{~d} y-\left(\alpha^{2}-1\right) \alpha^{-2} \int_{\Omega_{3}} b u_{1 \alpha} v \mathrm{~d} y
\end{aligned}
$$

and to the inequality

$$
\left\|u_{1}\right\|_{A, \Omega_{1}}^{2}=\int_{\Omega_{1}} \nabla^{\star} G \cdot \nabla u_{1} \mathrm{~d} x \leq c_{A b}^{-1 / 2}|G|_{1, \Omega_{1}}\left\|u_{1}\right\|_{A, \Omega_{1}},
$$

we can estimate

$$
\begin{aligned}
\left|a_{\Omega_{3}}\left(u_{3}-u_{1 \alpha}, v\right)\right| & \leq|v|_{1, \Omega_{3}}\left|G-G_{1 \alpha}\right|_{1, \Omega_{3}}+\left(\alpha^{2}-1\right) \alpha^{-2} b\left\|u_{1 \alpha}\right\|_{0, \Omega_{3}}\|v\|_{0, \Omega_{3}} \\
& \leq\|v\|_{1, \Omega_{3}}\left(\left|G-G_{1 \alpha}\right|_{1, \Omega_{3}}+\left(\alpha^{2}-1\right) b\left\|u_{1}\right\|_{0, \Omega_{1}}\right) \\
& \leq\|v\|_{1, \Omega_{3}}\left(\left|G-G_{1 \alpha}\right|_{1, \Omega_{3}}+\left(\alpha^{2}-1\right) \sqrt{b}\left\|u_{1}\right\|_{A, \Omega_{1}}\right) \\
& \leq\|v\|_{1, \Omega_{3}}\left(\left|G-G_{1 \alpha}\right|_{1, \Omega_{3}}+\left(\alpha^{2}-1\right) \sqrt{b} c_{A b}^{-1 / 2}|G|_{1, \Omega_{1}}\right) .
\end{aligned}
$$

Before we make the next step in estimating (4.10), let us formulate Lemma 4.4, which takes its motivation from $[\mathrm{M}]$.

Lemma 4.4. Let $\varphi \in L^{1}\left(\Omega_{3}\right) \cap C\left(\Omega_{3}\right)$ be a nonnegative function, and let $\varepsilon_{0}=$ $(\alpha-1) d_{0}, d_{0}=\sup _{x \in \Omega_{1}}\|x\|_{\mathbb{R}^{2}}$. Then

$$
\int_{\Omega_{1}}\left(\int_{x}^{\alpha x} \varphi(z) \mathrm{d} z\right) \mathrm{d} x \leq \varepsilon_{0} \int_{\Omega_{3}} \varphi(x) \mathrm{d} x .
$$


Proof. We follow an idea which can be found in the proof of [M, Lemma 1.4.6].

We reformulate the integral on the left-hand side. To this end we define the function $\varepsilon(x)=(\alpha-1)\|x\|_{\mathbb{R}^{2}}$. Its value at $x$ is equal to the length of the segment $(x, \alpha x)$. It holds $\varepsilon_{0} \leq(\alpha-1) \operatorname{diam} \Omega_{1}$. We can define $\varphi(x)=0$ if $x \notin \Omega_{3}$ and estimate

$$
\begin{aligned}
\int_{\Omega_{1}}\left(\int_{x}^{\alpha x} \varphi(z) \mathrm{d} z\right) \mathrm{d} x & =\int_{\Omega_{1}}\left(\int_{0}^{\varepsilon(x)} \varphi\left(x+t \frac{x}{\|x\|_{\mathbb{R}^{2}}}\right) \mathrm{d} t\right) \mathrm{d} x \\
& \leq \int_{\Omega_{1}}\left(\int_{0}^{\varepsilon_{0}} \varphi\left(x+t \frac{x}{\|x\|_{\mathbb{R}^{2}}}\right) \mathrm{d} t\right) \mathrm{d} x \equiv I .
\end{aligned}
$$

To estimate $I$, we define $\phi(x, t) \equiv \varphi\left(x+t \frac{x}{\|x\|_{\mathbb{R}^{2}}}\right), I_{\phi}(t) \equiv \int_{\Omega_{1}} \phi(x, t) \mathrm{d} x$, and circles $c(r)$ with the center at the origin and the radius $r \in\left(0, d_{0}\right)$. Then

$$
\begin{aligned}
I_{\phi}(t) & =\int_{0}^{d_{0}} \int_{c(r)} \phi(s, t) \mathrm{d} s \mathrm{~d} r \leq \int_{0}^{d_{0}} \int_{c(r+t)} \phi(s, 0) \mathrm{d} s \mathrm{~d} r \\
& \leq \int_{0}^{d_{0}+t} \int_{c(r)} \phi(s, 0) \mathrm{d} s \mathrm{~d} r \leq \int_{\Omega_{3}} \phi(x, 0) \mathrm{d} x .
\end{aligned}
$$

The first inequality is due to the fact that $\phi(\cdot, t)$ and $\phi(\cdot, 0)$ have identical values along $c(r)$ and $c(r+t)$, respectively, but the length of the circles is different. Thus

$$
I=\int_{0}^{\varepsilon_{0}} I_{\phi}(t) \mathrm{d} t \leq \int_{0}^{\varepsilon_{0}}\left(\int_{\Omega_{3}} \varphi(x) \mathrm{d} x\right) \mathrm{d} t=\varepsilon_{0} \int_{\Omega_{3}} \varphi(x) \mathrm{d} x .
$$

Lemma 4.5. Let $G \in H^{2}\left(\Omega_{3}\right)$ and $d_{0}$ be defined as in Lemma 4.4. Then

$$
\left|G-G_{1 \alpha}\right|_{1, \Omega_{3}}^{2} \leq 2 \alpha^{2}(\alpha-1)^{2}\left(d_{0}|G|_{2, \Omega_{3}}+|G|_{1, \Omega_{1}}\right)^{2} .
$$

Proof. We will write $G_{\alpha}$ instead of $G_{1 \alpha}$. First we suppose $G \in C^{\infty}(B)$. Then $G_{\alpha}$ is also smooth. As regards $G_{\alpha}$, we will distinguish between differentiating with respect to $y=\left(y_{1}, y_{2}\right) \in \Omega_{3}$ and $x=\left(x_{1}, x_{2}\right) \in \Omega_{1}$, i.e., if we confine ourselves to $x_{1}, y_{1}$ only,

$$
G_{\alpha, y_{1}}(\widetilde{y}) \equiv \frac{\partial G_{\alpha}(\widetilde{y})}{\partial y_{1}}=\frac{\partial G_{\alpha}(y(\widetilde{x}))}{\partial y_{1}}=\frac{1}{\alpha} \frac{\partial G(\widetilde{x})}{\partial x_{1}}, \quad \widetilde{y}=\alpha \widetilde{x} .
$$

If fixed $G$ and nonexpanded $\Omega_{1}$ are considered, then we can write

$$
\left.\frac{\partial G(x)}{\partial x_{1}}\right|_{x=\widetilde{x}}=\left.\frac{\partial G(y)}{\partial y_{1}}\right|_{y=\widetilde{x}}, \quad \widetilde{x} \in \Omega_{1} \subset \Omega_{3} .
$$

It is sufficient to show only an estimate for $G_{, y_{1}}$ and $G_{\alpha, y_{1}}$. We use $\varepsilon_{0}$ as in Lemma 4.4. Making use of the above equalities, we obtain

$$
\begin{aligned}
G_{, y_{1}}(\widetilde{y})- & G_{\alpha, y_{1}}(\widetilde{y})=G_{, y_{1}}(\alpha \widetilde{x})-\frac{1}{\alpha} G_{, x_{1}}(\widetilde{x}) \\
& =G_{, y_{1}}(\alpha \widetilde{x})-G_{, y_{1}}(\widetilde{x})+\frac{\alpha-1}{\alpha} G_{, x_{1}}(\widetilde{x}), \quad \widetilde{y} \in \Omega_{3}, \widetilde{x} \in \Omega_{1}, \widetilde{y}=\alpha \widetilde{x} .
\end{aligned}
$$


Thus we have

$$
\begin{aligned}
I \equiv & \int_{\Omega_{3}}\left(G_{, y_{1}}-G_{\alpha, y_{1}}\right)^{2} \mathrm{~d} y \\
=\int_{\Omega_{1}}\left[\left(G_{, y_{1}}(\alpha x)-G_{, y_{1}}(x)\right)^{2}\right. & \\
& \quad+2\left(G_{, y_{1}}(\alpha x)-G_{, y_{1}}(x)\right) G_{, x_{1}}(x) \frac{\alpha-1}{\alpha} \\
& \left.\quad+\left(G_{, x_{1}}(x) \frac{\alpha-1}{\alpha}\right)^{2}\right] \alpha^{2} \mathrm{~d} x \\
& \quad
\end{aligned}
$$

Taking into account

$$
\left|G_{, y_{1}}(\alpha x)-G_{, y_{1}}(x)\right| \leq \int_{x}^{\alpha x}\left|\nabla G_{, y_{1}}(z)\right| \mathrm{d} z
$$

the Schwarz inequality, and Lemma 4.4, we deduce

$$
\begin{aligned}
I_{1} & \leq \int_{\Omega_{1}}\left(\int_{x}^{\alpha x}\left|\nabla G_{, y_{1}}\right| \mathrm{d} z\right)^{2} \alpha^{2} \mathrm{~d} x \\
& \leq \alpha^{2} \int_{\Omega_{1}} \varepsilon_{0}\left(\int_{x}^{\alpha x}\left|\nabla G_{, y_{1}}\right|^{2} \mathrm{~d} z\right) \mathrm{d} x \leq \alpha^{2} \varepsilon_{0}^{2} \int_{\Omega_{3}}\left|\nabla G_{, y_{1}}\right|^{2} \mathrm{~d} x .
\end{aligned}
$$

By the Schwarz inequality and $\alpha>1$,

$$
\left|I_{2}\right| \leq 2 \alpha^{2}(\alpha-1) \varepsilon_{0}\left(\int_{\Omega_{3}}\left|\nabla G_{, y_{1}}\right|^{2} \mathrm{~d} x\right)^{1 / 2}\left(\int_{\Omega_{1}} G_{, x_{1}}^{2} \mathrm{~d} x\right)^{1 / 2} .
$$

We estimate $I_{3}$ by

$$
I_{3}=(\alpha-1)^{2} \int_{\Omega_{1}} G_{, x_{1}}^{2} \mathrm{~d} x \leq \alpha^{2}(\alpha-1)^{2} \int_{\Omega_{1}} G_{, x_{1}}^{2} \mathrm{~d} x .
$$

Combining (4.13)-(4.16) and the definition of $\varepsilon_{0}$, we get

$$
I \leq \alpha^{2}(\alpha-1)^{2}\left[d_{0}\left(\int_{\Omega_{3}}\left|\nabla G_{, y_{1}}\right|^{2} \mathrm{~d} x\right)^{1 / 2}+\left(\int_{\Omega_{1}} G_{, x_{1}}^{2} \mathrm{~d} x\right)^{1 / 2}\right]^{2} .
$$

We can infer a similar estimate for $G_{, y_{2}}-G_{\alpha, y_{2}}$ and, consequently, (4.11) for smooth $G$. Since smooth functions are dense in $H^{2}\left(\Omega_{3}\right)$, the proof is finished.

Lemma 4.6. Under the assumptions of Lemma 4.5,

$$
\left\|u_{3}-u_{1 \alpha}\right\|_{A, \Omega_{3}} \leq(\alpha-1) \theta
$$

where

$$
\theta=\sqrt{2} c_{A b}^{-1 / 2} \alpha\left(d_{0}|G|_{2, \Omega_{3}}+|G|_{1, \Omega_{1}}\right)+\sqrt{b} c_{A b}^{-1}(\alpha+1)|G|_{1, \Omega_{1}} .
$$

Proof. Applying (4.10) with $v=u_{3}-u_{1 \alpha}$ and (4.11), we estimate

$$
\begin{array}{r}
\left\|u_{3}-u_{1 \alpha}\right\|_{A, \Omega_{3}}^{2} \leq\left\|u_{3}-u_{1 \alpha}\right\|_{1, \Omega_{3}}\left(\left|G-G_{1 \alpha}\right|_{1, \Omega_{3}}+\left(\alpha^{2}-1\right) \sqrt{b} c_{A b}^{-1 / 2}|G|_{1, \Omega_{1}}\right) \\
\leq c_{A b}^{-1 / 2}\left\|u_{3}-u_{1 \alpha}\right\|_{A, \Omega_{3}}\left[\sqrt{2} \alpha(\alpha-1)\left(d_{0}|G|_{2, \Omega_{3}}+|G|_{1, \Omega_{1}}\right)\right. \\
\left.+\left(\alpha^{2}-1\right) \sqrt{b} c_{A b}^{-1 / 2}|G|_{1, \Omega_{1}}\right] .
\end{array}
$$

Cancelling $\left\|u_{3}-u_{1 \alpha}\right\|_{A, \Omega_{3}}$ on both sides, we get (4.17). 
We can start to estimate the right-hand side of (4.5).

Lemma 4.7. Under the assumptions of Lemma 4.5,

$$
\left\|u_{1}\right\|_{A, \Omega_{1}}^{2}-\left\|u_{3}\right\|_{A, \Omega_{3}}^{2} \leq(\alpha-1) \theta c_{A b}^{-1 / 2}\left(|G|_{1, \Omega_{1}}+|G|_{1, \Omega_{3}}\right) .
$$

Proof. The inequality in (4.8), the triangle inequality, and (4.17) lead to

$$
\begin{aligned}
\left\|u_{1}\right\|_{A, \Omega_{1}} \leq\left\|u_{1 \alpha}\right\|_{A, \Omega_{3}} & \leq\left\|u_{1 \alpha}-u_{3}\right\|_{A, \Omega_{3}}+\left\|u_{3}\right\|_{A, \Omega_{3}} \\
& \leq(\alpha-1) \theta+\left\|u_{3}\right\|_{A, \Omega_{3}} .
\end{aligned}
$$

Since both $u_{1}$ and $u_{3}$ solve (3.1) on $\Omega_{1}$ and $\Omega_{3}$, respectively, we get

$$
\left\|u_{i}\right\|_{A, \Omega_{i}}^{2} \leq\left\|u_{i}\right\|_{1, \Omega_{i}}|G|_{1, \Omega_{i}} \leq c_{A b}^{-1 / 2}\left\|u_{i}\right\|_{A, \Omega_{i}}|G|_{1, \Omega_{i}}, \quad i=1,3 .
$$

Transferring $\left\|u_{3}\right\|_{A, \Omega_{3}}$ to the left-hand side of (4.18), cancelling $\left\|u_{i}\right\|_{A, \Omega_{i}}$ on both sides of (4.19), and plugging the quantities into

$$
\left\|u_{1}\right\|_{A, \Omega_{1}}^{2}-\left\|u_{3}\right\|_{A, \Omega_{3}}^{2}=\left(\left\|u_{1}\right\|_{A, \Omega_{1}}-\left\|u_{3}\right\|_{A, \Omega_{3}}\right)\left(\left\|u_{1}\right\|_{A, \Omega_{1}}+\left\|u_{3}\right\|_{A, \Omega_{3}}\right),
$$

we finish the proof.

Theorem 4.1. Let $\nabla G \in\left[L^{\infty}\left(\Omega_{13}\right)\right]^{2}$ and $G \in H^{2}\left(\Omega_{3}\right)$. Then

$$
\begin{aligned}
& \left\|u_{2}-u_{1}\right\|_{A, \Omega_{1}}^{2} \\
& \quad \leq(\alpha-1) c_{A b}^{-1 / 2}\left[\theta\left(|G|_{1, \Omega_{1}}+|G|_{1, \Omega_{3}}\right)+2(\alpha+1) \operatorname{meas} \Omega_{1} c_{A b}^{-1 / 2}\|\nabla G\|_{\infty, \Omega_{13}}^{2}\right],
\end{aligned}
$$

where $\theta$ is defined in Lemma 4.6.

Proof. By virtue of (4.5), Lemma 4.7, Lemma 4.1, and meas $\Omega_{13}=\left(\alpha^{2}-1\right)$ meas $\Omega_{1}$,

$$
\begin{aligned}
& \left\|u_{2}-u_{1}\right\|_{A, \Omega_{1}}^{2} \leq\left\|u_{1}\right\|_{A, \Omega_{1}}^{2}-\left\|u_{3}\right\|_{A, \Omega_{3}}^{2}+\left\|u_{12}\right\|_{A, \Omega_{12}}^{2}+\left\|u_{23}\right\|_{A, \Omega_{23}}^{2} \\
& \quad \leq(\alpha-1) \theta c_{A b}^{-1 / 2}\left(|G|_{1, \Omega_{1}}+|G|_{1, \Omega_{3}}\right)+2\left(\alpha^{2}-1\right) \text { meas } \Omega_{1} c_{A b}^{-1}\|\nabla G\|_{\infty, \Omega_{13}}^{2} .
\end{aligned}
$$

Remark 4.2. As in the previous section, $a_{\Omega}$ can be replaced by $a_{\Omega}^{0}$. Then all results remain valid provided that we also substitute the first seminorm for the Sobolev norm and consider factor spaces $H^{1} / P$ instead of the Sobolev spaces $H^{1}$ at appropriate places. Some parts would be even simpler as a result of $b=0$ (see e.g., (4.8) and Lemma 4.6).

Remark 4.3. Let us emphasize that the estimate in Theorem 4.1 does not depend on the particular choice of $u_{2}$, i.e., $\widetilde{H}$. The estimate covers all possibilities discussed in the introductory part of Section 4.

Remark 4.4. The same upper bound as in Theorem 4.1 can be applied to estimate $\left\|u_{3}-u_{2}\right\|_{A, \Omega_{2}}^{2}$. Indeed, by Lemma 4.2 (cf. (4.5))

$$
\begin{aligned}
& \left\|u_{3}-u_{2}\right\|_{A, \Omega_{2}}^{2} \leq\left\|u_{3}-\left(u_{2}, u_{23}\right)\right\|_{A, \Omega_{3}}^{2} \\
& =\left\|u_{2}\right\|_{A, \Omega_{2}}^{2}+\left\|u_{23}\right\|_{A, \Omega_{23}}^{2}-\left(\left(u_{2}, u_{23}\right)-u_{3}+u_{3}, u_{3}\right)_{A, \Omega_{3}} \\
& \quad \leq\left\|u_{1}\right\|_{A, \Omega_{1}}^{2}+\left\|u_{12}\right\|_{A, \Omega_{12}}^{2}+\left\|u_{23}\right\|_{A, \Omega_{23}}^{2}-\left\|u_{3}\right\|_{A, \Omega_{3}}^{2},
\end{aligned}
$$

and the last part coincides with the right-hand side of (4.5). 
Domains with the Lipschitz boundary. We would like to generalize the idea utilized in the previous subsection to not necessarily starshaped domains, namely to the class of domains with the Lipschitz boundary.

Having a starshaped domain $\Omega_{1}$, we can easily "blow it up" to get a superdomain $\Omega_{3} \supset \bar{\Omega}_{2} \supset \Omega_{2} \supset \bar{\Omega}_{1}$. The following lemma shows that we can blow up even more general domains, though the mapping doing this job is not as simple as before.

Lemma 4.8. Let $\Omega_{1}$ be a domain with the Lipschitz boundary. Then a parameter $\varepsilon_{0}$ can be found such that for any $\varepsilon, 0<\varepsilon \leq \varepsilon_{0}$, a smooth mapping $\varkappa_{\varepsilon}$ and a domain $\Omega_{3}^{\varepsilon}$ exist and it holds $\Omega_{3}^{\varepsilon}=\varkappa_{\varepsilon}\left(\Omega_{1}\right), \bar{\Omega}_{1} \subset \Omega_{3}^{\varepsilon}$. Moreover, $\operatorname{dist}\left(x, \Omega_{1}\right) \leq \varepsilon C$ for any $x \in \Omega_{3}^{\varepsilon} \backslash \Omega_{1}$, where $C$ is an arbitrary constant greater than one.

Proof. The idea of the proof comes from the technique used in [N1] and [N2]. The proof is outlined as follows.

The Lipschitz boundary can be locally defined as a graph of a Lipschitz function. This can be closely approximated by a smooth function. By means of such functions certain local mappings will be defined. These together with the partition of unity will lead to the mapping $\varkappa_{\varepsilon}$.

Let $S$ be a global Cartesian coordinate system for $\Omega_{1}$. According to the definition of the Lipschitz boundary (see [NH] or, equivalently, [N2]), there exist real numbers $\alpha>0$ and $\beta>0$ such that for each $x_{0} \in \partial \Omega_{1}$ we can rotate and translate $S$ to get a local Cartesian system $S_{x_{0}}^{\prime}$ having the following properties: the origin of $S_{x_{0}}^{\prime}$ coincides with $x_{0}$; a Lipschitz continuous function $\omega$ exists in $S_{x_{0}}^{\prime}$ such that it maps the segment $(-\alpha, \alpha)$ onto a part of the boundary $\partial \Omega_{1}$ and, moreover, the sets defined in $S_{x_{0}}^{\prime}$ as

$$
\begin{aligned}
& M_{<}^{\prime}=\left\{\widehat{x}=\left(\widehat{x}_{1}, \widehat{x}_{2}\right): \widehat{x}_{1} \in(-\alpha, \alpha) \text { and } \omega\left(\widehat{x}_{1}\right)-\beta<\widehat{x}_{2}<\omega\left(\widehat{x}_{1}\right)\right\}, \\
& M_{>}^{\prime}=\left\{\widehat{x}=\left(\widehat{x}_{1}, \widehat{x}_{2}\right): \widehat{x}_{1} \in(-\alpha, \alpha) \text { and } \omega\left(\widehat{x}_{1}\right)<\widehat{x}_{2}<\omega\left(\widehat{x}_{1}\right)+\beta\right\}
\end{aligned}
$$

are subsets of $\Omega_{1}$ and $\mathbb{R}^{2} \backslash \bar{\Omega}_{1}$, respectively.

In the system $S_{x_{0}}^{\prime}$, we fix the point $\left(0, \inf _{-\alpha<\widehat{x}_{1}<\alpha} \omega\left(\widehat{x}_{1}\right)-\beta\right)$ as the origin of a new Cartesian coordinate system $S_{x_{0}}$ parallel to $S_{x_{0}}^{\prime}$. Let us again denote the function describing a part of $\partial \Omega_{1}$ by $\omega$.

Our final goal is to construct a continuously differentiable mapping $\varkappa$. To this end, we first define two sets by means of the coordinate system $S_{x_{0}}$ :

$$
\begin{aligned}
& M_{\omega}=\left\{y: y_{1} \in(-\alpha, \alpha) \text { and } 0<y_{2}<\omega\left(y_{1}\right)\right\}, \\
& M_{\omega}^{\beta}=\left\{y: y_{1} \in(-\alpha, \alpha) \text { and } 0<y_{2}<\omega\left(y_{1}\right)+\beta\right\} .
\end{aligned}
$$

We can suppose that $\beta$ is small enough to ensure $M_{\omega} \subset \Omega_{1}$.

We choose a small parameter $\varepsilon, 0<\varepsilon \ll \beta$, and approximate $\omega$ by an infinitely smooth positive function $\eta$. According to [A, Lemma 2.18], we can assume $\|\omega-\eta\|_{\infty,(-\alpha, \alpha)} \leq \zeta$, where $0<\zeta \ll \varepsilon$ is an arbitrary small positive value.

We set up a smooth mapping $\kappa_{\varepsilon}$ defined on $M_{\omega}^{\beta}$ as

$$
\kappa_{\varepsilon}(y)=\left(y_{1}, y_{2}+\frac{\varepsilon}{\eta\left(y_{1}\right)} \gamma\left(y_{1}, y_{2}\right)\right), \quad y=\left(y_{1}, y_{2}\right) \in M_{\omega}^{\beta},
$$

where $\gamma$ is a smooth function on $M_{\omega}^{\beta}$, nondecreasing in $y_{2}$, and such that $\gamma\left(y_{1}, 0\right)=0$ and $\gamma\left(y_{1}, \eta\left(y_{1}\right)\right)=\eta\left(y_{1}\right)$ for $y_{1} \in(-\alpha, \alpha)$. Function $\gamma$ is not specified in detail and offers a possibility to appropriately adjust $\kappa_{\varepsilon}$ to some needs which become apparent later. 
It holds that $\bar{M}_{\omega} \subset \kappa_{\varepsilon}\left(M_{\omega}\right)$ because $\zeta$ is small. The set $\kappa_{\varepsilon}\left(M_{\omega}\right)$ covers $\partial \Omega_{1}$ in the vicinity of $x_{0}$.

The set $\partial \Omega_{1}$ is compact and therefore can be covered by a finite number, say $N$, of domains $U_{i}, i=1,2, \ldots, N$, defined by the same manner as $M_{\omega}^{\beta}$ in (4.20). Similarly, we consider functions $\eta_{i}$, mappings $\gamma_{i}$ and define smooth mappings $\kappa_{\varepsilon}^{i}$ on $U_{i}$ (see (4.21)). Adding just one appropriate domain $U_{N+1} \subset \Omega_{1}, \bar{U}_{N+1} \subset \Omega_{1}$, we get a family $\Theta$ of open sets covering $\Omega_{1}$, i.e., $\bigcup_{i=1}^{N+1} U_{i} \supset \bar{\Omega}_{1}$. We can assume that any point $x \in \Omega_{1}$ belongs at most to two sets $U_{j}, U_{k}, j, k \in\{1,2, \ldots, N\}, j \neq k$, and possibly also to $U_{N+1}$. Adjusting $\beta$, we can also suppose that if $\left(U_{j} \cap U_{k}\right) \backslash \Omega_{1} \neq \emptyset$, then $\left(U_{j} \cap U_{k}\right) \cap \Omega_{1} \neq \emptyset$, i.e., if $U_{j}$ and $U_{k}$ intersect then the intersection is not a proper subset of $\mathbb{R}^{2} \backslash \Omega_{1}$.

We define a $C^{\infty}$-partition of unity for $\Omega_{1}$ subordinate to $\Theta$ and denote its functions by $\varphi_{i}, i=1,2, \ldots, N+1$ (see [N2]).

We have much freedom in defining $\gamma_{i}$ so we can suppose that a constant $\delta$, $\beta>\delta \gg \varepsilon$, exists such that

$$
\Omega_{\delta}=\left\{x \in \Omega_{1}: \operatorname{dist}\left(x, \partial \Omega_{1}\right) \leq \delta\right\} \subset \bigcup_{i=1}^{N} U_{i}
$$

and that $\gamma_{i}(y)=0$ if $y \in U_{i} \backslash \Omega_{\delta / 2}$, where $\Omega_{\delta / 2}$ is defined by the parameter $\delta / 2$ used in (4.22) instead of $\delta$. We assume $\Omega_{\delta} \cap U^{N+1}=\emptyset$.

To get a mapping in the global coordinate system $S$, we transform mappings $\kappa_{\varepsilon}^{i}$ from the local coordinate systems to the global coordinate system, and we denote the transformed mappings by $\tilde{\varkappa}_{\varepsilon}^{i}$. We define $\tilde{\varkappa}_{\varepsilon}^{N+1}$ on $U_{N+1}$ as an identity mapping.

Finally, we introduce mappings $\varkappa_{\varepsilon}^{i}, i=1, \ldots, N+1$, defined as follows: $\varkappa_{\varepsilon}^{i}(x)=$ $\varphi_{i}(x) \widetilde{\varkappa}_{\varepsilon}^{i}(x)$ if $x \in U_{i}$. By the properties of $\Theta$, each $\varkappa_{\varepsilon}^{i}$ is a smooth mapping and has its support contained in $U_{i}$.

Summing up $\varkappa_{\varepsilon}^{i}$ and restricting the domain of definition to $\Omega_{1}$, we get a smooth mapping

$$
\varkappa_{\varepsilon}(x)=\sum_{i=1}^{N+1} \varkappa_{\varepsilon}^{i}(x), \quad x \in \Omega_{1} .
$$

The mapping $\varkappa_{\varepsilon}$ is equal to the identity mapping on a subdomain of $\Omega_{1}$ and, if $\zeta$, $\varepsilon$, and $\gamma_{i}$ are properly chosen, it maps the boundary layer $\Omega_{\delta / 2}$ of $\Omega_{1}$ onto a larger layer containing $\partial \Omega_{1}$.

We can label $\varepsilon$ as $\varepsilon_{0}$ and repeat the above steps for a new parameter $\varepsilon, 0<\varepsilon<\varepsilon_{0}$. Two possibilities can happen. Either $\zeta, \eta_{i}, \gamma_{i}$ can remain unchanged or we have to adjust them appropriately.

We see that the constant $C$ depends on $\zeta$ and $\eta_{i}$. But the smaller $\zeta$ the closer $C$ to one we can get.

Lemma 4.9. Let $\varkappa_{\varepsilon}$ be the mapping from Lemma 4.8 with a parameter $\varepsilon>0$. Then

$$
\varkappa_{\varepsilon}(x)=\left(y_{1}, y_{2}\right), \quad y_{i}=x_{i}+e_{i}^{\varepsilon}\left(x_{1}, x_{2}\right), i=1,2, \quad x \in \Omega_{1},
$$

where $e_{i}^{\varepsilon}$ is a smooth function. Positive constants $C_{e}, C_{e}^{\prime}$ independent of $\varepsilon$ exist such that

$$
\left\|e_{i}^{\varepsilon}\right\|_{\infty, \Omega_{1}} \leq \varepsilon C_{e}, \quad\left\|\frac{\partial e_{i}^{\varepsilon}}{\partial x_{j}}\right\|_{\infty, \Omega_{1}} \leq \varepsilon C_{e}^{\prime}, \quad i, j=1,2
$$


As a consequence, $\varkappa_{\varepsilon}$ is a one-to-one mapping if $\varepsilon$ is small, i.e., $\varepsilon C_{e} \ll 1, \varepsilon C_{e}^{\prime} \ll 1$.

Proof. We can take up the proof of Lemma 4.8. According to (4.21), any mapping $\tilde{\varkappa}_{\varepsilon}^{i}, i=1,2, \ldots, N+1$, is a small perturbation of identity, i.e., $\tilde{\varkappa}_{\varepsilon}^{i}(x)=x+e_{\varepsilon}^{i}(x)$, $e_{\varepsilon}^{N+1}=0$. The sum $\sum_{i=1}^{N+1} \varphi(x)$ equals 1 if $x \in \Omega_{1}$ so

$$
\varkappa_{\varepsilon}(x)=x+\sum_{i=1}^{N} \varphi_{i}(x) e_{\varepsilon}^{i}(x)=x+e^{\varepsilon}(x), \quad e^{\varepsilon}(x)=\left(e_{1}^{\varepsilon}(x), e_{2}^{\varepsilon}(x)\right) .
$$

By the properties of the partition of unity, (4.21), and Lemma 4.8,

$$
\left\|e_{j}^{\varepsilon}\right\|_{\infty, \Omega_{1}} \leq \varepsilon C, \quad j=1,2,
$$

and $C_{e}$ is close to one if $\zeta, \eta_{i}$ and $\gamma_{i}$ are properly chosen.

Let us focus on the constant $C_{e}^{\prime}$. It depends on $\varphi_{i}, \eta_{i}, \gamma_{i}$, and their derivatives. The partition of unity is fixed, therefore functions $\varphi_{i}$ as well as overlapping parts of $U_{i}$ are fixed too.

The derivative of $\eta_{i}$ can be bounded independently of $i$ and $\zeta$ because $\omega$ has a fixed Lipschitz constant along the boundary $\partial \Omega$; i.e., $\omega$ can be approached by a sequence of smooth functions the Lipschitz constant of which is uniformly bounded, but possibly different.

Also, functions $\gamma_{i}$ though indirectly dependent on $\varepsilon$ can be constructed in such a way that their first derivatives are bounded independently of $\varepsilon$.

We infer that functions $\varphi_{i}$ as well as $\eta_{i}$ and $\gamma_{i}$ are smooth with first derivatives bounded in $\Omega_{1}$ independently of $\varepsilon$. Thus an $\varepsilon C_{e}^{\prime}$ bound is guaranteed.

Remark 4.5. Due to the assumption made in the proof of Lemma $4.8, \varkappa_{\varepsilon}$ maps a $\delta / 2$-layer along $\partial \Omega_{1}$ onto a $(\delta / 2+\varepsilon)$-layer containing $\partial \Omega_{1}$. The role $\varepsilon$ plays here is similar to that of $\alpha-1$ in the starshaped domain case. We have a lot of freedom in choosing $\gamma_{i}$ and thus ensuring both the invertibility of $\varkappa_{\varepsilon}$ and a reasonable value of $C_{e}^{\prime}$.

By Lemma $4.9, \varkappa_{\varepsilon}$ can be constructed as a small perturbation of the identity mapping. As a consequence, we can immediately formulate the statement of Lemma 4.9 for the inverse mapping $\varkappa_{\varepsilon}^{-1}$.

Lemma 4.10. Let $\varkappa_{\varepsilon}$ be the mapping from Lemma 4.9 restricted to $\Omega_{1}$, and $\Omega_{3}^{\varepsilon}=$ $\varkappa_{\varepsilon}\left(\Omega_{1}\right)$. Then $\varkappa_{\varepsilon}^{-1}(y)=\left(x_{1}, x_{2}\right)$, where $x_{i}=y_{i}+g_{i}^{\varepsilon}\left(y_{1}, y_{2}\right), i=1,2$, maps $\Omega_{3}^{\varepsilon}$ onto $\Omega_{1}$. Also, positive constants $C_{g}, C_{g}^{\prime}$, independent of $\varepsilon$, exist such that $\left\|g_{i}^{\varepsilon}\right\|_{\infty, \Omega_{3}} \leq$ $\varepsilon C_{g},\left\|\partial g_{i}^{\varepsilon} / \partial x_{j}\right\|_{\infty, \Omega_{3}} \leq \varepsilon C_{g}^{\prime}$.

In the next parts, we will follow the ideas presented in the course of the already performed analysis of starshaped domains. In contrast to it, we will face formulae complicated by some additional terms, the order of which, however, will be equal to $\varepsilon$.

We again deal with domains $\Omega_{1}, \Omega_{2}$, and, omitting the superscript $\varepsilon$,

$$
\Omega_{3}=\left\{y \in \mathbb{R}^{2}: \exists x \in \Omega_{1} \quad y=\varkappa_{\varepsilon}(x)\right\},
$$

where $\varkappa_{\varepsilon}$ is the mapping from Lemma $4.9, \bar{\Omega}_{1} \subset \Omega_{2} \subset \bar{\Omega}_{2} \subset \Omega_{3} \subset \bar{\Omega}_{3} \subset B$, and $\Omega_{2}$ can be $\mathrm{N}$-unstable.

Focusing on $\varkappa_{\varepsilon}^{-1}: \Omega_{3} \rightarrow \Omega_{1}$ and a differentiable function $w(x)=w\left(\varkappa_{\varepsilon}^{-1}(y)\right)=$ $v(y)$, we evaluate quantities corresponding to (4.6) applied to $\varkappa_{\varepsilon}^{-1}$; i.e., the roles of 
$x_{i}$ and $y_{i}$ are interchanged:

$$
\begin{aligned}
D & =1+\frac{\partial g_{1}}{\partial y_{1}}+\frac{\partial g_{2}}{\partial y_{2}}+\frac{\partial g_{1}}{\partial y_{1}} \frac{\partial g_{2}}{\partial y_{2}}-\frac{\partial g_{2}}{\partial y_{1}} \frac{\partial g_{1}}{\partial y_{2}}, \\
\frac{\partial w}{\partial x_{1}} & =\left[\frac{\partial v}{\partial y_{1}}\left(1+\frac{\partial g_{2}}{\partial y_{2}}\right)-\frac{\partial v}{\partial y_{2}} \frac{\partial g_{2}}{\partial y_{1}}\right] / D \\
\frac{\partial w}{\partial x_{2}} & =\left[\frac{\partial v}{\partial y_{2}}\left(1+\frac{\partial g_{1}}{\partial y_{1}}\right)-\frac{\partial v}{\partial y_{1}} \frac{\partial g_{1}}{\partial y_{2}}\right] / D .
\end{aligned}
$$

We can write $\nabla_{x} w=\left(\nabla_{y} v+M_{g} \nabla_{y} v\right) / D$, where the $2 \times 2$ matrix $M_{g}$ comprises partial derivatives of $g_{1}$ and $g_{2}$. Let us notice that $D>0$ if $\varepsilon$ is sufficiently small.

We introduce $u_{1 \varepsilon}(y)=u_{1 \varepsilon}\left(\varkappa_{\varepsilon}(x)\right)=u_{1}(x)$ and transfer $a_{\Omega_{1}}\left(u_{1}, w\right)$ from $\Omega_{1}$ to $\Omega_{3}$

$$
\begin{aligned}
\int_{\Omega_{1}}( & \left.A \nabla u_{1} \cdot \nabla w+b u_{1} w \mathrm{~d} x\right) \\
= & \int_{\Omega_{3}} A\left(\nabla_{y} u_{1 \varepsilon}+M_{g} \nabla_{y} u_{1 \varepsilon}\right) \cdot\left(\nabla_{y} v+M_{g} \nabla_{y} v\right)|D| / D^{2} \mathrm{~d} y \\
& \quad+\int_{\Omega_{3}} b u_{1 \varepsilon} v|D| \mathrm{d} y \\
= & \int_{\Omega_{3}} A \nabla u_{1 \varepsilon} \cdot \nabla v D^{-1} \mathrm{~d} y+\widehat{a}\left(g ; u_{1 \varepsilon}, v\right)+\int_{\Omega_{3}} b u_{1 \varepsilon} v D \mathrm{~d} y \\
= & a_{\Omega_{3}}\left(u_{1 \varepsilon}, v\right)+a_{g}\left(u_{1 \varepsilon}, v\right) .
\end{aligned}
$$

The forms $\widehat{a}$ and $a_{g}$ comprise terms with $M_{g}$ and $1-D$, it is $D^{-1}=1+(1-D) / D$.

On the basis of Lemma 4.10,

$$
\begin{aligned}
\left|a_{g}\left(u_{1 \varepsilon}, v\right)\right| & \leq \varepsilon \hat{C}_{1}\left\|u_{1 \varepsilon}\right\|_{1, \Omega_{3}}\|v\|_{1, \Omega_{3}} \leq \varepsilon \hat{C}_{1} \tilde{C}_{1}|G|_{1, \Omega_{1}}\|v\|_{1, \Omega_{3}} \\
& \leq \varepsilon C_{1}|G|_{1, \Omega_{1}}\|v\|_{1, \Omega_{3}}, \quad v \in H^{1}\left(\Omega_{3}\right),
\end{aligned}
$$

because $\left\|u_{1 \varepsilon}\right\|_{1, \Omega_{3}} \leq c_{A b}^{-1}|G|_{1, \Omega_{1}}+\left\|u_{1}\right\|_{1, \Omega_{1}} \mathcal{O}(\varepsilon) \leq \tilde{C}_{1}|G|_{1, \Omega_{1}}$ as can be seen from (4.23), (2.6), and (2.3).

Denoting $G_{1 \varepsilon}(y)=G_{1}\left(\varkappa_{\varepsilon}^{-1}(y)\right)=G_{1}(x),\left.G_{1} \equiv G\right|_{\Omega_{1}}$, we have

$$
\begin{aligned}
\int_{\Omega_{1}} & \nabla_{x}^{\star} G_{1} \cdot \nabla_{x} w \mathrm{~d} x \\
& =\int_{\Omega_{3}}\left(\nabla^{\star} G_{1 \varepsilon}+M_{g}^{\star} \nabla^{\star} G_{1 \varepsilon}\right) \cdot\left(\nabla v+M_{g} \nabla v\right)|D| / D^{2} \mathrm{~d} y \\
& =\int_{\Omega_{3}} \nabla^{\star} G_{1 \varepsilon} \cdot \nabla v \mathrm{~d} y+E_{g}\left(G_{1 \varepsilon}, v\right),
\end{aligned}
$$

where $M_{g}^{\star}$ comprises permuted elements of $M_{g}$ and $E_{g}\left(G_{1 \varepsilon}, \cdot\right)$ is a continuous linear form on $H^{1}\left(\Omega_{3}\right)$. Again, a positive constant $C_{2}$ independent of $\varepsilon$ exists such that

$$
\left|E_{g}\left(G_{1 \varepsilon}, v\right)\right| \leq \varepsilon C_{2}|v|_{1, \Omega_{3}} .
$$

By (4.24) and (4.26), $u_{1 \varepsilon} \in H^{1}\left(\Omega_{3}\right)$ solves the equation

$$
a_{\Omega_{3}}\left(u_{1 \varepsilon}, v\right)+a_{g}\left(u_{1 \varepsilon}, v\right)=\int_{\Omega_{3}} \nabla^{\star} G_{1 \varepsilon} \cdot \nabla v \mathrm{~d} y+E_{g}\left(G_{1 \varepsilon}, v\right) \quad \forall v \in H^{1}\left(\Omega_{3}\right) .
$$


Let us remark that $\varkappa_{\varepsilon}$ and $\varkappa_{\varepsilon}^{-1}$ transform $H^{1}\left(\Omega_{1}\right)$ onto $H^{1}\left(\Omega_{3}\right)$ and vice versa, respectively, because both mappings are sufficiently smooth quasi-isometric mappings (see [M Section 1.1.7]).

Our aim is to derive an estimate assessing the difference between $u_{1 \varepsilon}$ and $u_{3}$, the solution of (2.6) on $\Omega_{3}$, cf. (4.10). We start with (see (4.25), (4.27))

$$
\begin{aligned}
& \left|a_{\Omega_{3}}\left(u_{3}-u_{1 \varepsilon}, v\right)\right| \\
& \quad=\left|\int_{\Omega_{3}} \nabla^{\star}\left(G-G_{1 \varepsilon}\right) \cdot \nabla v \mathrm{~d} y+a_{g}\left(u_{1 \varepsilon}, v\right)-E_{g}\left(G_{1 \varepsilon}, v\right)\right| \\
& \quad \leq\|v\|_{1, \Omega_{3}}\left(\left|G-G_{1 \varepsilon}\right|_{1, \Omega_{3}}+\varepsilon C_{1}|G|_{1, \Omega_{1}}+\varepsilon C_{2}\right) .
\end{aligned}
$$

We are at the point of estimating $\left|G-G_{1 \varepsilon}\right|_{1, \Omega_{3}}$, i.e., we need to generalize Lemma 4.5. To this end we put two additional assumptions on $\varkappa_{\varepsilon}$. First, we suppose onwards that any two points $x$ and $\varkappa_{\varepsilon}(x), x \in \Omega_{1}$, can be connected by a straight segment lying in $\Omega_{3}$. Second, as $\varkappa_{\varepsilon}$ are rather unspecified mappings, we have to assume that a generalization of Lemma 4.4 holds, i.e., if $\varphi \in L^{1}\left(\Omega_{3}\right) \cap C\left(\Omega_{3}\right)$ is a nonnegative function, then

$$
\int_{\Omega_{1}} \int_{x}^{\varkappa(x)} \varphi(z) \mathrm{d} z \mathrm{~d} x \leq \varepsilon C \int_{\Omega_{3}} \varphi(x) \mathrm{d} x,
$$

where $C>0$ does not depend on $\varepsilon$.

Lemma 4.11. Let $G \in H^{2}\left(\Omega_{3}\right)$ and $G_{1 \varepsilon}$ be the composite of $\left.G\right|_{\Omega_{1}}$ and $\varkappa_{\varepsilon}^{-1}$ from Lemma 4.10. Then

$$
\left|G-G_{1 \varepsilon}\right|_{1, \Omega_{3}} \leq \varepsilon C_{3}\left(|G|_{1, \Omega_{1}}+|G|_{2, \Omega_{3}}\right),
$$

where $C_{3}$ is a positive constant independent of $\varepsilon$ and $G$.

Proof. We will write $G_{\varepsilon}$ and $\varkappa$ instead of $G_{1 \varepsilon}$ and $\varkappa_{\varepsilon}$, respectively. First, we assume that $G \in C^{\infty}(B)$. Then $G_{\varepsilon}$ is also smooth on $\Omega_{3}$.

By Lemma 4.9 and (4.23) adapted to $\varkappa$, we observe, if $\widetilde{y}=\left(\widetilde{y}_{1}, \widetilde{y}_{2}\right)=\varkappa(\widetilde{x})$ and

$$
\widetilde{D}=1+\frac{\partial e_{1}}{\partial x_{1}}+\frac{\partial e_{2}}{\partial x_{2}}+\frac{\partial e_{1}}{\partial x_{1}} \frac{\partial e_{2}}{\partial x_{2}}-\frac{\partial e_{2}}{\partial x_{1}} \frac{\partial e_{1}}{\partial x_{2}},
$$

that

$$
\begin{aligned}
G_{\varepsilon, y_{1}}(\widetilde{y}) \equiv & \frac{\partial G_{\varepsilon}(\widetilde{y})}{\partial y_{1}} \\
= & {\left[\frac{\partial G(\widetilde{x})}{\partial x_{1}}\left(1+\frac{\partial e_{2}(\widetilde{x})}{\partial x_{2}}\right)-\frac{\partial G(\widetilde{x})}{\partial x_{2}} \frac{\partial e_{2}(\widetilde{x})}{\partial x_{1}}\right] / \widetilde{D}(\widetilde{x}) } \\
= & \frac{\partial G(\widetilde{x})}{\partial x_{1}}+\frac{\partial G(\widetilde{x})}{\partial x_{1}} \frac{1-\widetilde{D}(\widetilde{x})}{\widetilde{D}(\widetilde{x})} \\
& +\left[\frac{\partial G(\widetilde{x})}{\partial x_{1}} \frac{\partial e_{2}(\widetilde{x})}{\partial x_{2}}-\frac{\partial G(\widetilde{x})}{\partial x_{2}} \frac{\partial e_{2}(\widetilde{x})}{\partial x_{1}}\right] / \widetilde{D}(\widetilde{x}) \\
= & \left.\frac{\partial G(x)}{\partial x_{1}}\right|_{x=\widetilde{x}}+\psi_{1}(\widetilde{x})=\left.\frac{\partial G(y)}{\partial y_{1}}\right|_{y=\widetilde{x}}+\psi_{1}(\widetilde{x})
\end{aligned}
$$

where function $\psi_{1}$ comprises all terms with partial derivatives of $e_{1}, e_{2}$. The last equality is (4.12) in essence. 
Thus we get

$$
\left|G_{, y_{1}}(\widetilde{y})-G_{\varepsilon, y_{1}}(\widetilde{y})\right| \leq\left|G_{, y_{1}}(\widetilde{y})-G_{, y_{1}}(\widetilde{x})\right|+\left|\psi_{1}(\widetilde{x})\right| .
$$

Connecting $\widetilde{x}$ and $\widetilde{y}$ by a straight segment, we estimate

$$
\left|G_{, y_{1}}(\widetilde{y})-G_{, y_{1}}(\widetilde{x})\right| \leq \int_{\widetilde{x}}^{\varkappa(\widetilde{x})}\left|\nabla G_{, x_{1}}(z)\right| \mathrm{d} z=\psi_{2}(\widetilde{x}) .
$$

Integrating (4.31) over $\Omega_{3}$, we infer

$$
\begin{aligned}
\int_{\Omega_{3}}\left(G_{, y_{1}}-G_{\varepsilon, y_{1}}\right)^{2} \mathrm{~d} y & \leq \int_{\Omega_{1}}\left(\left|\psi_{1}(x)\right|+\psi_{2}(x)\right)^{2}|\widetilde{D}(x)| \mathrm{d} x \\
& =\int_{\Omega_{1}}\left(\psi_{1}^{2}+2\left|\psi_{1} \psi_{2}\right|+\psi_{2}^{2}\right)|\widetilde{D}| \mathrm{d} x \equiv I_{1}+I_{2}+I_{3} .
\end{aligned}
$$

By (4.30) and Lemma 4.9

$$
I_{1}=\int_{\Omega_{1}} \psi_{1}^{2}|\widetilde{D}| \mathrm{d} x \leq \varepsilon^{2} \widehat{C} \int_{\Omega_{1}}|\nabla G|^{2} \mathrm{~d} x=\varepsilon^{2} \widehat{C}|G|_{1, \Omega_{1}}^{2},
$$

where $\widehat{C}>0$ is a constant independent of $G$ and $\varepsilon$ if $0<\varepsilon<\varepsilon_{0}$ and $\varepsilon_{0}$ is a small parameter.

On the basis of (4.29) we get

$$
\begin{aligned}
I_{3} & =\int_{\Omega_{1}}\left(\int_{x}^{\varkappa(x)}\left|\nabla G_{, x_{1}}\right| \mathrm{d} z\right)^{2}|\widetilde{D}| \mathrm{d} x \\
& \leq \varepsilon \widetilde{C} \int_{\Omega_{1}}\left(\int_{x}^{\varkappa(x)}\left|\nabla G_{, x_{1}}\right|^{2} \mathrm{~d} z\right) \mathrm{d} x \leq \varepsilon^{2} \widetilde{C} \int_{\Omega_{3}}\left|\nabla G_{x_{1}}\right|^{2} \mathrm{~d} x,
\end{aligned}
$$

where a positive constant $\widetilde{C}$ does not depend on $\varepsilon, 0<\varepsilon \leq \varepsilon_{0}$, and $G$.

Finally, applying (4.33), (4.34), and the Schwarz inequality, we infer

$$
\begin{aligned}
I_{2} & =2 \int_{\Omega_{1}}\left|\psi_{1} \psi_{2}\right||\widetilde{D}| \mathrm{d} x \\
& \leq 2 C\left(\int_{\Omega_{1}} \psi_{1}^{2} \mathrm{~d} x\right)^{1 / 2}\left(\int_{\Omega_{1}} \psi_{2}^{2} \mathrm{~d} x\right)^{1 / 2} \leq \varepsilon^{2} C^{\prime}|G|_{1, \Omega_{1}}\left|G_{, x_{1}}\right|_{1, \Omega_{3}},
\end{aligned}
$$

where, again, $C^{\prime}>0$ is independent of $\varepsilon$ and $G$.

Plugging (4.33)-(4.35) into (4.32), we arrive at

$$
\int_{\Omega_{3}}\left(G_{, y_{1}}-G_{\varepsilon, y_{1}}\right)^{2} \mathrm{~d} y \leq \varepsilon^{2} C_{3}^{2}\left(|G|_{1, \Omega_{1}}+\left|G_{, x_{1}}\right|_{1, \Omega_{3}}\right)^{2} / 2
$$

The estimates for $G_{, y_{2}}-G_{\varepsilon, y_{2}}$ can be derived in a similar way. Referring to the density of smooth functions in $H^{2}\left(\Omega_{3}\right)$, we finish the proof.

Applying Lemma 4.11 to (4.28) with $v=u_{3}-u_{1 \varepsilon}$, we generalize Lemma 4.6.

Lemma 4.12. If $G \in H^{2}\left(\Omega_{3}\right)$, then

$$
\left\|u_{3}-u_{1 \varepsilon}\right\|_{A, \Omega_{3}} \leq \varepsilon \widehat{\theta},
$$

where $\widehat{\theta}=c_{A b}^{-1 / 2}\left[\left(C_{1}+C_{3}\right)|G|_{1, \Omega_{1}}+C_{3} \mid G_{2, \Omega_{3}}+C_{2}\right]$. Constants $C_{1}, C_{2}$, and $C_{3}$ come from (4.25), (4.27), and Lemma 4.11.

We also adjust Lemma 4.1. 
Lemma 4.13. Let us assume both $\nabla G \in\left[L^{\infty}\left(\Omega_{13}\right)\right]^{2}$ and meas $\Omega_{13} \leq \varepsilon C_{4} c_{A b} / 2$, $C_{4}$ is a positive constant independent of $\varepsilon$. Then

$$
\left\|u_{12}\right\|_{A, \Omega_{12}}^{2}+\left\|u_{23}\right\|_{A, \Omega_{23}}^{2} \leq \varepsilon C_{4}\|\nabla G\|_{\infty, \Omega_{13}}^{2} .
$$

We need some analogy to the inequality in (4.8). By (4.24) we have

$$
\left\|u_{1}\right\|_{A, \Omega_{1}}^{2}=\left\|u_{1 \varepsilon}\right\|_{A, \Omega_{3}}^{2}+a_{g}\left(u_{1 \varepsilon}, u_{1 \varepsilon}\right) .
$$

Let us consider a domain $\Omega_{2}, \bar{\Omega}_{1} \subset \Omega_{2} \subset \bar{\Omega}_{2} \subset \Omega_{3}$. Proceeding as in the starshaped domain case, we can formulate a generalization of Theorem 4.1.

Theorem 4.2. Let $\Omega_{1}, \Omega_{2}$, and $\Omega_{3}$ be the domains introduced in previous paragraphs, and let $u_{1}$ and $u_{2}$ be the respective solution to (3.1) on $\Omega_{1}, H=H^{1}\left(\Omega_{1}\right)$, and $\Omega_{2}, H=\widetilde{H}$. Let $\varkappa_{\varepsilon}$ be the mapping from Lemma 4.9, $\Omega_{3}=\varkappa_{\varepsilon}\left(\Omega_{1}\right)$. Assume $\nabla G \in\left[L^{\infty}\left(\Omega_{13}\right)\right]^{2}$ and $G \in H^{2}\left(\Omega_{3}\right)$. Then

$$
\left\|u_{2}-u_{1}\right\|_{A, \Omega_{1}}^{2} \leq \varepsilon C,
$$

where a positive constant $C$ depends on $|G|_{1, \Omega_{1}},|G|_{1, \Omega_{3}},|G|_{2, \Omega_{3}},\|\nabla G\|_{\infty, \Omega_{13}}$, and constants $c_{A b}, \tilde{C}_{1}, C_{1}, C_{2}, C_{3}$, and $C_{4}$, but is independent of $\varepsilon, 0<\varepsilon \leq \varepsilon_{0}$, if $\varepsilon_{0}$ is sufficiently small.

Proof. By (4.38), the triangle inequality, (4.25), and (4.36),

$$
\begin{aligned}
\left\|u_{1}\right\|_{A, \Omega_{1}}^{2} & =\left\|u_{1 \varepsilon}\right\|_{A, \Omega_{3}}^{2}+a_{g}\left(u_{1 \varepsilon}, u_{1 \varepsilon}\right) \\
& \leq\left(\left\|u_{1 \varepsilon}-u_{3}\right\|_{A, \Omega_{3}}+\left\|u_{3}\right\|_{A, \Omega_{3}}\right)^{2}+\varepsilon C_{1}|G|_{1, \Omega_{1}}\left\|u_{1 \varepsilon}\right\|_{1, \Omega_{3}} \\
& \leq \varepsilon^{2} \widehat{\theta}^{2}+2 \varepsilon \widehat{\theta}\left\|u_{3}\right\|_{A, \Omega_{3}}+\left\|u_{3}\right\|_{A, \Omega_{3}}^{2}+\varepsilon C_{1} \tilde{C}_{1}|G|_{1, \Omega_{1}}^{2} .
\end{aligned}
$$

We estimate $2 \varepsilon \widehat{\theta}\left\|u_{3}\right\|_{A, \Omega_{3}}$ by means of $|G|_{1, \Omega_{3}}$ and get

$$
\left\|u_{1}\right\|_{A, \Omega_{1}}^{2}-\left\|u_{3}\right\|_{A, \Omega_{3}}^{2} \leq \varepsilon^{2} \widehat{\theta}^{2}+2 \varepsilon \widehat{\theta} c_{A b}^{-1 / 2}|G|_{1, \Omega_{3}}+\varepsilon C_{1} \tilde{C}_{1}|G|_{1, \Omega_{1}}^{2} .
$$

To finish the proof, we plug (4.37) and (4.39) into (4.5):

$$
\begin{aligned}
\left\|u_{2}-u_{1}\right\|_{A, \Omega_{1}}^{2} \leq \varepsilon^{2} \widehat{\theta}^{2} & +2 \varepsilon \widehat{\theta} c_{A b}^{-1 / 2}|G|_{1, \Omega_{3}} \\
& +\varepsilon C_{1} \tilde{C}_{1}|G|_{1, \Omega_{1}}^{2}+\varepsilon C_{4}\|\nabla G\|_{\infty, \Omega_{13}}^{2} \leq \varepsilon C,
\end{aligned}
$$

where $C>0$ depends on $G$.

An analogy to Remark 4.4 is also valid.

Let us again emphasize that the estimate of $\left\|u_{2}-u_{1}\right\|_{A, \Omega_{1}}$ and $\left\|u_{3}-u_{2}\right\|_{A, \Omega_{2}}$ depend neither on the particular domain $\Omega_{2}$ nor its stability status, provided that $\Omega_{2}$ is approximated from inside and outside by $\Omega_{1}$ and $\Omega_{3}$, respectively. As a consequence, the estimate applies also to solutions $u_{m}$ mentioned in the introductory paragraphs of Section 4.

\section{Stability of $\Omega$ Under general Convergence $\Omega_{n} \rightarrow \Omega$}

We will show that if $\Omega$ is $\mathrm{N}$-stable with respect to monotone convergence $\Omega_{n} \nearrow \Omega, \Omega_{n} \searrow \Omega$, then it is also stable (in a natural sense) with respect to a general convergence $\Omega_{n} \rightarrow \Omega$.

Let $\partial \Omega=\partial \bar{\Omega}$ and $\left\{\Omega_{n}\right\}_{n=1}^{\infty}$ be a sequence of domains with Lipschitz boundary such that $\Omega_{n} \rightarrow \Omega$ in the set sense. 
We can construct sequences $\left\{\Omega_{n}^{\uparrow}\right\}_{n=1}^{\infty}$ and $\left\{\Omega_{n}^{\downarrow}\right\}_{n=1}^{\infty}$ such that $\partial \Omega_{n}^{\uparrow}, \partial \Omega_{n}^{\downarrow}$ are Lipschitz or even smooth, $\bar{\Omega}_{n}^{\uparrow} \subset \Omega_{n} \subset \bar{\Omega}_{n} \subset \Omega_{n}^{\downarrow}$, and $\Omega_{n}^{\uparrow} \nearrow \Omega, \Omega_{n}^{\downarrow} \searrow \Omega$. The spaces $H^{\uparrow}, H^{\downarrow}$ and functions $u_{G}, u^{G}$ are defined via these sequences (see Section 3).

Let $\left\{u_{n}^{\uparrow}\right\}_{n=1}^{\infty}$ and $\left\{u_{n}^{\downarrow}\right\}_{n=1}^{\infty}$ be the sequences of the solutions of (3.1) on $\Omega_{n}^{\uparrow}$ and $\Omega_{n}^{\downarrow}$, respectively, $n=1,2, \ldots$.

Theorem 5.1. Let $\Omega$ be stable with respect to the Neumann boundary value problem and monotone sequences of domains, i.e., $H^{\uparrow}=H^{\downarrow}$ and $u_{G}=u^{G}$. Then

$$
\lim _{n \rightarrow \infty}\left\|u_{n}^{\downarrow}-u_{n}\right\|_{A, \Omega_{n}}=0=\lim _{n \rightarrow \infty}\left\|u_{n}^{\uparrow}-u_{n}\right\|_{A, \Omega_{n}^{\uparrow}} .
$$

Proof. We wish to benefit from (4.5) and Remark 4.4. To this end we identify $\Omega_{n}^{\uparrow}, \Omega_{n}$ and $\Omega_{n}^{\downarrow}$ with $\Omega_{1}, \Omega_{2}$ and $\Omega_{3}$, respectively (see Section 4 ). We denote the respective solutions of (4.1) by $u_{n}^{12}$ and $u_{n}^{23}$.

Let us notice that by (4.1) and (2.3) $\left\|u_{n}^{i}\right\|_{A, \Omega_{i}} \leq C, i=12,23$, where $C$ is a positive constant independent of $n$, cf. the beginning of the proof of Lemma 3.1, for instance.

By Remark 4.4 and (4.1)

$$
\begin{aligned}
\left\|u_{n}^{\downarrow}-u_{n}\right\|_{A, \Omega_{n}}^{2} & \leq\left\|u_{n}^{\uparrow}\right\|_{A, \Omega_{n}^{\uparrow}}^{2}-\left\|u_{n}^{\downarrow}\right\|_{A, \Omega_{n}^{\downarrow}}^{2}+\left\|u_{n}^{12}\right\|_{A, \Omega_{n} \backslash \Omega_{n}^{\uparrow}}^{2}+\left\|u_{n}^{23}\right\|_{A, \Omega_{n}^{\downarrow} \backslash \Omega_{n}}^{2} \\
& \leq\left\|u_{n}^{\uparrow}\right\|_{A, \Omega_{n}^{\uparrow}}^{2}-\left\|u_{n}^{\downarrow}\right\|_{A, \Omega_{n}^{\downarrow}}^{2}+2 C|G|_{1, \Omega_{n}^{\downarrow} \backslash \Omega_{n}^{\uparrow}} .
\end{aligned}
$$

The right-hand side of the inequality tends to zero by virtue of Lemma 3.2, Lemma 3.3 (see (3.16)) and $\lim _{n \rightarrow \infty} \operatorname{meas}\left(\Omega_{n}^{\downarrow} \backslash \Omega_{n}^{\uparrow}\right)=0$. This and (4.5) also proves the other limit.

\section{ExAmple, APPLICATIONS, AND CONCLUSIONS}

Let us go back to Example 1.1 presented in the Introduction.

Based on the theory expounded in previous sections, the proposed approach to Neumann boundary value problems on uncertain domains consists in approximating the uncertain domain $\Omega$ by known domains $\Omega_{\text {low }}$ and $\Omega_{\text {up }}, \bar{\Omega}_{\text {low }} \subset \Omega \subset \bar{\Omega} \subset \Omega_{\text {up }}$, in setting the BVP with the boundary condition formulated in the nonclassic way elaborated in Section 2, in solving the BVP on $\Omega_{\text {low }}$ and $\Omega_{\mathrm{up}}$, respectively, and in applying estimates based on (4.5). Though a simple idea, it is not easy to bring it to life.

Figure 1 shows the original pixel domain in 256 levels of the gray color. Setting the threshold for white color to 123, we produced Figure 2 (left). Choices 63 and 190 lead to Figure 3 (left) and (right), respectively. We can see that the domain in Figure 3 (right) is embedded into the domain in Figure 2 (left), and this domain
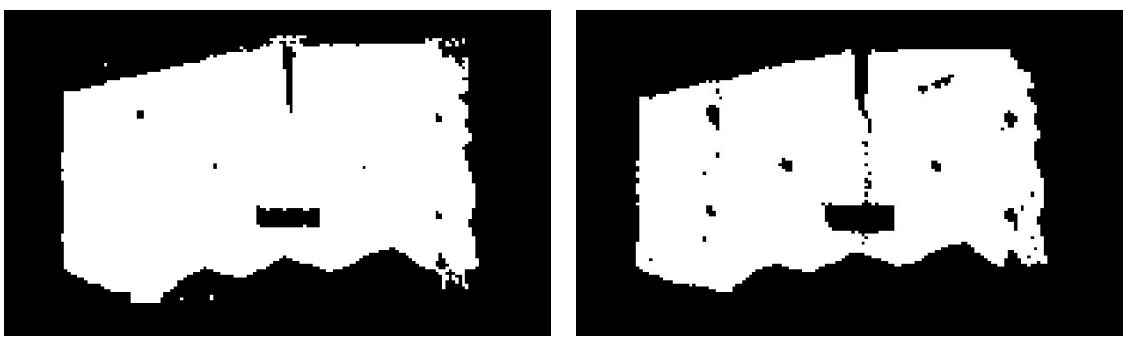

Figure 3. Postprocessing: Low threshold (left), high threshold (right) 
is further embedded to the largest domain depicted in Figure 3 (left). We can also observe deterioration of the images. The domain in Figure 2 (left) seems to be quite acceptable, whereas white pixels in Figure 3 (left) do not create a connected set. Figure 3 (right) shows a connected white set but its connectivity is far more multiple than in Figure 2 (left).

The question arises of how to choose threshold values. The bigger the difference between them, the larger the difference between respective white areas, and the greater the amount of uncertainty taken into consideration. Also, threshold values that are too low or too high would force the domain's pixels to turn into background pixels due to uneven contrast and brightness or noise superimposed onto the basic signal.

A rule of thumb could be to define a function describing the dependence of the total white area on the threshold value. Experience shows that such a function has a rapid decay for low and high thresholds and a slow decay in between. Values, where the slope of the function starts and ends to be moderate, seem to be a good choice to define $\Omega_{\text {low }}$ and $\Omega_{\text {up }}$.

If we suppose that the digitalized domain is connected, then by observing the number of connected white sets implied by threshold settings we can also arrive at reasonable approximations of $\Omega$.

Both approaches can be combined and, moreover, we can add a two-pixel-wide white layer to our upper approximation of $\Omega$ to get a strengthened $\Omega_{\mathrm{up}}$. By adding a black layer, we can get a strengthened $\Omega_{\text {low }}$. According to experiments, details beneath 1.5 pixel size are almost invisible. That is why we suggest adding layers two pixels wide.

We can also introduce some calibration stemming from a comparison between measured properties of a real sample and results of a digital image based computation.

Another difficulty arises if we compare the physical domain $\Omega$ with its, possibly postprocessed, digital images. It can happen that we do not get an upper or lower estimate of $\Omega$ simply because all details (e.g., cracks, micro-holes, and thin projections) below the digital image resolution are invisible or merged with other sources of pollution and noise.

This implies that though we wish to take into account as much uncertainty as is possible and reasonable, we still must make some assumptions. Basically, we have to assume that the digital image is a good representation of $\Omega$ in the sense that a manipulation with digital data can deliver reasonable domains $\Omega_{\text {low }}$ and $\Omega_{\mathrm{up}}$ estimating $\Omega$ from inside and outside, respectively. The notion reasonable is vague but it certainly does not mean whole white and black rectangles we can always produce as certainly true upper and lower estimates.

Having $\Omega_{\text {low }}$ and $\Omega_{\text {up }}$, we can apply the presented theory. Let us remark that $\Omega_{\text {low }}$ and $\Omega_{\text {up }}$ need not be pixel domains. They can have a piecewise smooth boundary (cf. Figure 2 (right)) as requested by computational methods, say, the finite or boundary elements. Thus we lose, however, the mesh formed from pixels which could be directly used in calculations.

From the computational point of view of the finite element method, it is advantageous, if the coefficients of the equation are constant, to have $\Omega_{\text {low }}$ and $\Omega_{\text {up }}$ starshaped, $\Omega_{\text {up }}=\alpha \Omega_{\text {low }}, \alpha>1$, because in that case we can assemble only one stiffness matrix as the other depends on $\alpha$ in a simple way. 
If $\Omega_{\text {low }}$ and $\Omega_{\text {up }}$ are not starshaped, they differ by a layer of elements which, if properly numbered, will lead to two different stiffness matrices, the smaller being a block of the larger one. This speeds up direct solving of the system of linear algebraic equations. One can also expect that solutions on $\Omega_{\text {low }}$ and $\Omega_{\text {up }}$ will not differ much; i.e., that one solution can be used as a good initial guess in an iterative solver to get the other solution.

Acknowledgments. Data for pictures used in this paper were produced and partly postprocessed by V. Pascucci and M. Sadahiro from TICAM, the University of Texas at Austin. The authors wish to thank both of them for fruitful collaboration. The authors also thank the anonymous referees and Prof. Lars Wahlbin for comments leading to the improvement of the manuscript.

\section{REFERENCES}

[A] R. A. Adams, Sobolev Spaces, Academic Press, Inc., Orlando, 1975. MR 56:9247.

[B1] I. Babuška, Stability of domains with respect to basic problems in the theory of partial differential equations, mainly the theory of elasticity, I, Czechoslovak Math. J. 11 (86) (1961), 76-105 (Russian).

[B2] I. Babuška, Stability of domains with respect to basic problems in the theory of partial differential equations, mainly the theory of elasticity, II, Czechoslovak Math. J. 11 (86) (1961), 165-203 (Russian).

[BCL] C. Bajaj, E. Coyle, K.Lin, Arbitrary topology shape reconstruction from planar cross sections, Graph. Models and Image Process. 58 (1996), 524-543.

[BPHN] C. Bajaj, V. Pascucci, R. Holt, A. Netravali, Active countouring of images with physical A-splines, TICAM Rep No 99-03, University of Texas at Austin (1999).

[H] J. Hadamard, Lectures on Cauchy's Problem in Linear Partial Differential Equations, Yale Univ. Press, New Haven, CT, 1923, Reprinted, Dover, 1952. MR 14:474f

[HN] J. Haslinger, P. Neittaanmäki, Finite Element Approximation for Optimal Shape, Material and Topology Design, 2nd edition, John Wiley and Sons, Chichester, 1996. MR 97k:49002

[HCK] E. J. Haug, K. K. Choi, V. Komkov, Design Sensitivity Analysis of Structural Systems, Academic Press, Inc., Orlando, 1986. MR 89i:73061

[K] T. Kolsrud, Approximation by smooth functions in Sobolev spaces. A counterexample., Bull. Lond. Math. Soc. 13 (1981), 67-69. MR 83h:46052

[M] V. G. Maz'ja, Sobolev Spaces, Springer-Verlag, Berlin, 1985. MR 87g:46056

[N1] J. Nečas, Sur les domaines du type $\mathfrak{N}$, Czech. Math. J. 12 (1962), 274-287 (Russian).

[N2] J. Nečas, Les méthodes directes en théorie des équations elliptiques, Masson/Academia, Paris/ Prague, 1967.

[NH] J. Nečas, I. Hlaváček, Mathematical Theory of Elastic and Elasto-Plastic Bodies: An Introduction, Elsevier, Amsterdam, 1981. MR 82h:73002

[R] P. J. Rouche, Verification and Validation in Computational Science and Engineering, Hermosa Publishers, Albuquerke N.M., USA, 1998.

The University of Texas at Austin, TiCAM, Austin, Texas 78713

Mathematical Institute, Academy of Sciences of the Czech Republic, Žitná 25, 11567 Prague 1, Czech Republic

E-mail address: chleb@math.cas.cz 